\title{
The Methylcitrate Cycle is Required for Development and Virulence in the Rice Blast Fungus Pyricularia oryzae
}

\author{
Yuxin Yan, ${ }^{1}$ Huan Wang, ${ }^{1}$ Siyi Zhu, ${ }^{1}$ Jing Wang, ${ }^{1}$ Xiaohong Liu, ${ }^{2}$ Fucheng Lin, ${ }^{2}$ and Jianping Lu ${ }^{1, \dagger}$ \\ ${ }^{1}$ State Key Laboratory for Rice Biology, College of Life Sciences, Zhejiang University, Hangzhou 310058, Zhejiang Province, \\ China \\ ${ }^{2}$ State Key Laboratory for Rice Biology, Biotechnology Institute, Zhejiang University
}

Accepted 27 March 2019.

\begin{abstract}
The methylcitrate cycle metabolizes propionyl-CoA, a toxic metabolite, into pyruvate. Pyricularia oryzae (syn. Magnaporthe oryzae) is a phytopathogenic fungus that causes a destructive blast disease in rice and wheat. We characterized the essential roles of the methylcitrate cycle in the development and virulence of $P$. oryzae using functional genomics. In $P$. oryzae, the transcript levels of MCS1 and MCL1, which encode a 2-methylcitrate synthase and a 2-methylisocitrate lyase, respectively, were upregulated during appressorium formation and when grown on propionyl-CoA-producing carbon sources. We found that deletion of MCS1 and MCL1 inhibited fungal growth on media containing both glucose and propionate, and media using propionate or propionyl-CoA-producing amino acids (valine, isoleucine, methionine, and threonine) as the sole carbon or nitrogen sources. The $\Delta m c s 1$ mutant formed sparse aerial hyphae and did not produce conidia on complete medium (CM), while the $\Delta m c l 1$ mutant showed decreased conidiation. The aerial mycelium of $\Delta m c s 1$ displayed a lowered $\mathrm{NAD}^{+} / \mathrm{NADH}$ ratio, reduced nitric oxide content, and downregulated transcription of hydrophobin genes. $\Delta m c l 1$ showed reduced appressorium turgor, severely delayed plant penetration, and weakened virulence. Addition of acetate recovered the growth of the wild type and $\Delta m c s 1$ on medium containing both glucose and propionate and recovered the conidiation of both $\Delta m c s 1$ and $\Delta m c l 1$ on $\mathrm{CM}$ by reducing propionyl-CoA formation. Deletion of $M C L 1$ together with $I C L 1$, an isocitrate lyase gene in the glyoxylate cycle, greatly reduced the mutant's virulence as compared with the single-gene deletion mutants ( $\Delta i c l 1$ and $\Delta m c l 1)$. This experimental evidence provides important information about the role of the methylcitrate cycle in development and virulence of $P$. oryzae by detoxification of propionyl-CoA and 2-methylisocitrate.
\end{abstract}

Keywords: fungal development, fungus-plant interactions, mechanisms of pathogenicity

${ }^{\dagger}$ Corresponding author: J. Lu; jplu@zju.edu.cn

Funding: This work was supported by the National Natural Science Foundation of China (grants 31671975 and 31871908).

*The $\boldsymbol{e}$-Xtra logo stands for "electronic extra" and indicates that two supplementary figures and three supplementary tables are published online.

The author(s) declare no conflict of interest.

(c) 2019 The American Phytopathological Society
Propionyl-CoA is a metabolite produced by the degradation of propionate, amino acids (valine, isoleucine, methionine, and threonine) thymine, cholesterol, and odd-chain fatty acids (Graham and Eastmond 2002; Halarnkar and Blomquist 1989). In animals and some bacteria, propionyl-CoA is metabolized into succinyl-CoA via the methylmalonyl-CoA pathway and then enters the tricarboxylic acid (TCA) cycle (Halarnkar and Blomquist 1989; Peyraud et al. 2009). In fungi and some bacteria, propionyl-CoA is detoxified via the methylcitrate cycle (Pronk et al. 1994; Uchiyama et al. 1982). In the methylcitrate cycle (Fig. 1A), propionyl-CoA and oxaloacetate are condensed into methylcitrate by 2-methylcitrate synthase (Mcs), converted into 2-methyl-cis-aconitate by 2-methylcitrate dehydratase (Mcd), subsequently converted into 2-methylisocitrate by aconitase (Acn), and then cleaved into succinate and pyruvate by 2-methylisocitrate lyase (Mcl) (Uchiyama et al. 1982). Finally, succinate and pyruvate are transformed into oxaloacetate or other metabolites, respectively, via the TCA cycle or other metabolic pathways (Brock et al. 2000, 2002).

Propionyl-CoA, 2-methylcitrate, and methylioscitrate inhibit cell growth and are toxic to organisms. 2-Methylcitrate inhibits the cell growth of Salmonella enterica (Horswill et al. 2001). In Aspergillus nidulans, propionyl-CoA inhibits pyruvate dehydrogenase, ATP citrate lyase, and succinyl-CoA synthetase (Brock and Buckel 2004), and 2-methylisocitrate inhibits NADPdependent isocitrate (Brock 2005). Therefore, sodium propionate is widely used to inhibit fungal growth (Brock 2005; Brock and Buckel 2004). In humans, propionyl-CoA noncompetitively inhibits pyruvate dehydrogenase complex activity (Schwab et al. 2006), and 2-methylcitrate competitively inhibits citrate synthase, aconitase, nicotinamide adenine dinucleotide ${ }^{+}\left(\mathrm{NAD}^{+}\right)$and $\mathrm{NADP}^{+}$-linked isocitrate dehydrogenase, and phosphofructokinase (Cheema-Dhadli et al. 1975). Accumulation of propionyl-CoA, 2-methylcitrate, and metabolites of propionate oxidation led to mitochondrial dysfunction in propionic aciduria patients (Cheema-Dhadli et al. 1975; Schwab et al. 2006).

In A. nidulans, $\triangle m c s A$ and $\Delta m c l A$, mutants in which the 2methylcitrate synthase gene or 2-methylisocitrate lyase gene, respectively, was deleted could not utilize propionate as the sole carbon source, and propionate inhibited the growth of $\triangle m c l A$ in media containing glucose, glycerol, or acetate (Brock 2005; Brock et al. 2000). Accumulation of propionyl-CoA inhibited the synthesis of polyketides such as carcinogens, mycotoxins, and sterigmatocystin in A. nidulans and A. fumigatus (Brock and Buckel 2004; Maerker et al. 2005; Zhang et al. 2004). In A. fumigatus, $\triangle m c s A$ could not grow on medium using propionate as the sole carbon source, propionate inhibited the growth of $\triangle m c s A$ in media containing glucose, and the virulence 
A

Propionate

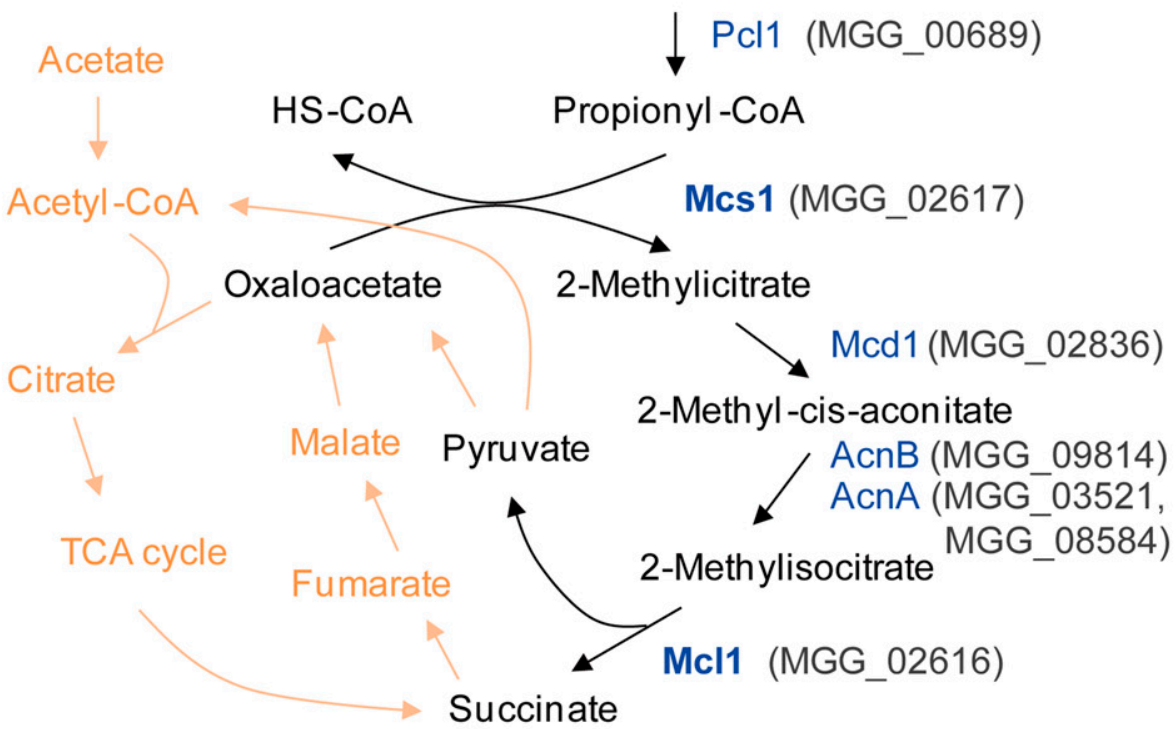

B
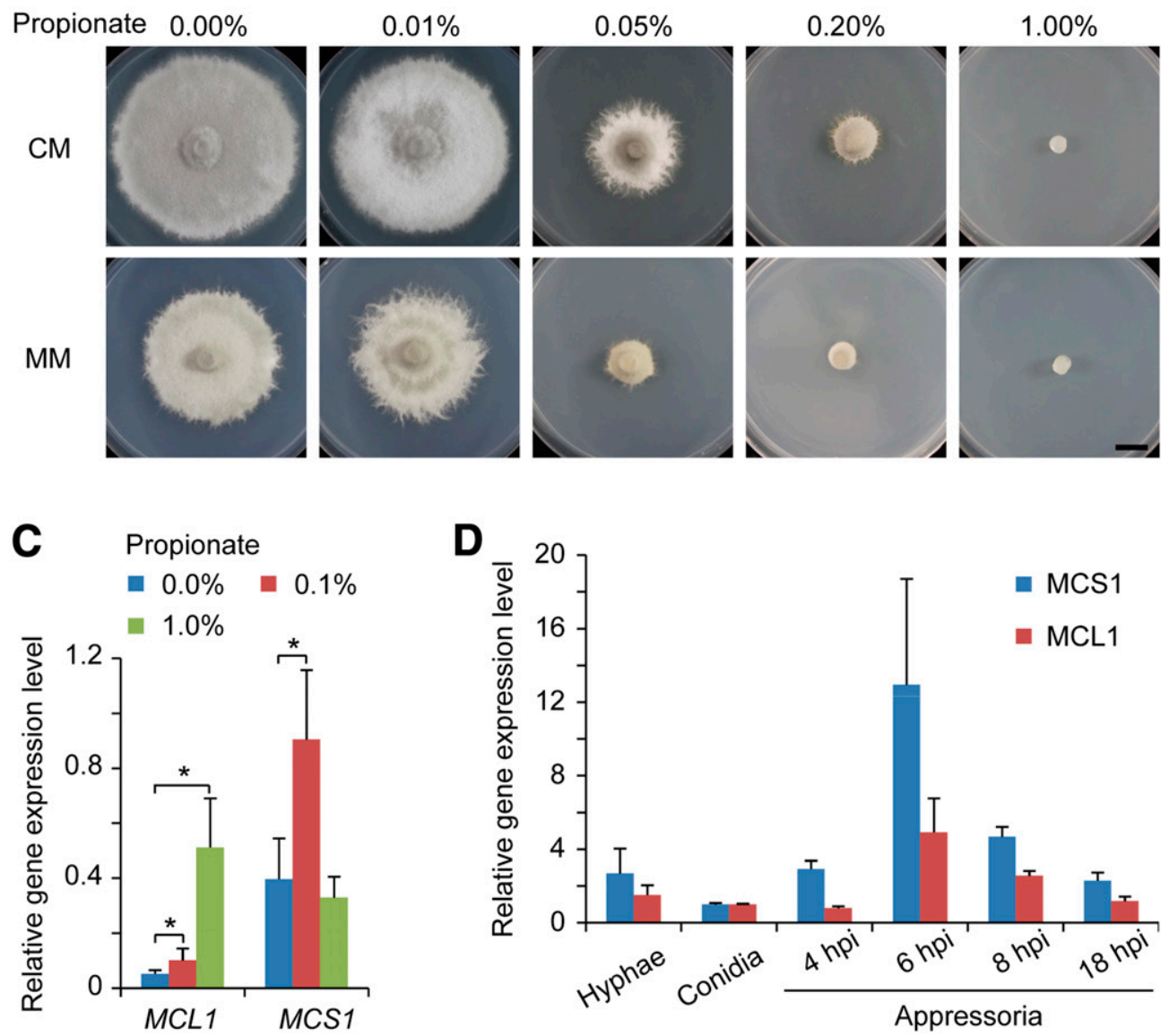

Fig. 1. Methylcitrate cycle in Pyricularia oryzae. A, Homologous proteins of the methylcitrate cycle in P. oryzae. AcnA and AcnB $=\operatorname{aconitases,~Mcd1~}=2-$ methylcitrate dehydratase, Mcl1 = 2-methylisocitrate lyase, Mcs1 = 2-methylcitrate synthase, and Pcl1 = propionate-CoA ligase. B, Propionate inhibits the growth of $P$. oryzae. The wild type was cultured on complete medium (CM) and minimal medium (MM) (1\% glucose) containing different concentrations of sodium propionate at $25^{\circ} \mathrm{C}$ for 9 days. Bar $=10 \mathrm{~mm}$. C, Relative expression levels of $M C S 1$ and $M C L 1$ in response to propionate. The wild type was cultured in $\mathrm{MM}$ containing different concentrations of sodium propionate at $25^{\circ} \mathrm{C}$ for $6 \mathrm{~h}$. Asterisks indicate significant differences between two columns as estimated by Tukey's honestly significant difference test, $P<0.05$. D, Relative expression levels of MCS1 and MCL1 in hyphae cultured in CM, conidia (as 1), and appressoria at $4,6,8$, and $18 \mathrm{~h}$ postinoculation. In $\mathrm{C}$ and $\mathrm{D}, \beta$-TUBULIN and $\alpha$-ACTIN were used as reference genes. 
of $\Delta m c s A$ in animals was reduced (Ibrahim-Granet et al. 2008; Maerker et al. 2005). In Gibberella zeae, the virulence of $\Delta m c l l$ on barley and wheat was also reduced (Lee et al. 2009). In Trichoderma atroviride, $\Delta i c l 2$, in which the 2-methylisocitrate lyase gene (ICL2) was deleted, could not utilize propionate as the sole carbon source, and showed reduced inhibition of Botrytis cinerea growth (Dubey et al. 2013).

Pyricularia oryzae (syn. Magnaporthe oryzae) is a filamentous ascomycete fungus that causes rice and wheat blast, diseases leading to serious crop loss around the world (Dean et al. 2012). Carbon metabolism plays important roles in the development and pathogenicity of $P$. oryzae. Lipid catabolism, the glyoxylate cycle, gluconeogenesis, and their regulatory pathways such as the PMK1 mitogen-activated protein kinase pathway, the cAMPdependent protein kinase A signaling pathway, and the carbon catabolite repression pathway are required for the production of appressorium turgor, appressorium-mediated penetration, and infection (Badaruddin et al. 2013; Cao et al. 2018; Fernandez et al. 2012; Thines et al. 2000; Wang et al. 2007). Deletion of many enzyme genes in carbohydrate metabolism and lipid metabolism, including a transketolase, Tkl1 (Fernandez et al. 2014); a glyoxylate aminotransferase, Agt1 (Bhadauria et al. 2012); an isocitrate lyase, Icl1 (Wang et al. 2003); a methylmalonatesemialdehyde dehydrogenase, Msdh (Norvienyeku et al. 2017); and glycerol-3-phosphate dehydrogenases Gpd1 and Gpd2 (Shi et al. 2018) all led to defects in fungal development and virulence. However, the role of the methylcitrate cycle, an important pathway involved in propionyl-CoA metabolism, in

\section{B}

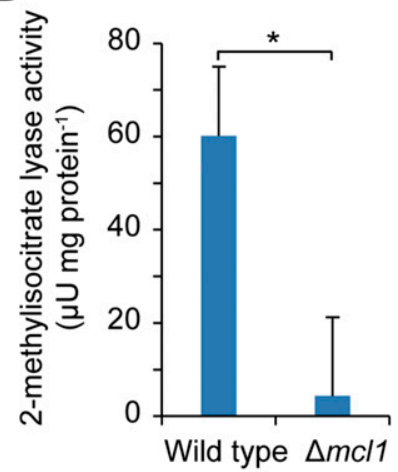

C

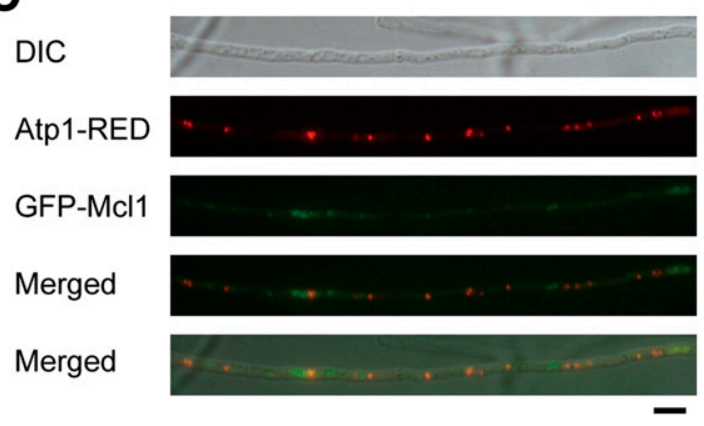

Fig. 2. Enzyme activity and subcellular localization assays. A, 2-Methylcitrate synthase activity in the hyphae of $\Delta m c s 1$. B, 2-Methylisocitrate lyase activity in the hyphae of $\Delta m c l 1$. Enzyme activity was shown in microunits per milligram of protein $\left(\mu \mathrm{U}\right.$ mg protein $\left.{ }^{-1}\right)$. Error bars represent standard deviation. Asterisks indicate significant differences between two columns as estimated by Tukey's honestly significant difference test, $P<0.05$. C, Subcellular localization of Mcl1 in the mitochondria of Pyricularia oryzae hyphae. Green fluorescent protein (GFP)-Mcl1 fusion proteins were colocalized with an $\alpha$ subunit of mitochondrial F1F0 ATP synthase Atp1. Bar $=10 \mu \mathrm{m}$ the development and pathogenicity of $P$. oryzae still remains unclear.

In this study, we characterized the functions of the methylcitrate cycle in $P$. oryzae by deleting a 2-methylcitrate synthase gene, $M C S 1$, and a 2-methylisocitrate lyase gene, MCL1. Using genetic and molecular analyses, we showed that the methylcitrate cycle is necessary for propionate metabolism, cellular redox state, nitric oxide (NO) production, growth, conidiation, appressorium turgor production, and virulence in the rice blast fungus.

\section{RESULTS}

Methylcitrate cycle in $P$. oryzae.

Propionate inhibited the growth of a $P$. oryzae wild-type strain cultured on complete medium (CM) and minimal medium (MM) containing $1 \%$ glucose (Fig. 1B). The depressing effect of propionate was dose dependent, and $1 \%$ propionate completely blocked the growth of the wild type on CM and $\mathrm{MM}$, suggesting that propionate is deleterious to this fungus. Propionate is metabolized via the methylcitrate cycle (Fig. 1A) and homologous proteins of the five enzymes in the methylcitrate cycle were found in $P$. oryzae by searching its genome against homologous proteins identified in other organisms (Fig. 1A; Supplementary Table S1). MCS1 (MGG_02617) encodes a 2-methylcitrate synthase and MCL1 (MGG_02616) encodes a 2-methylisocitrate lyase (Fig. 1A). MCS1 and MCL1 are adjacent genes on the genome. We measured the changes in transcript levels of MCS1 and MCL1 in response to propionate addition in MM. The expression of both $M C S 1$ and $M C L 1$ was significantly upregulated in MM with $0.1 \%$ propionate, whereas only the expression of $M C L 1$ continued to be upregulated in MM with $1.0 \%$ propionate (Fig. 1C). We then examined MCS1 and MCL1 expression levels in the hyphae cultured in CM, resting conidia, and developing appressoria, and found that the transcript levels of MCS1 and MCL1 increased during conidium germination and appressorium formation, reaching the highest in the appressoria at $6 \mathrm{~h}$ postinoculation (hpi) (Fig. 1D).

To identify the roles of the methylcitrate cycle in development and virulence of $P$. oryzae, we deleted MCL1 and MCS1 in the wild-type strain 70-15 (Supplementary Fig. S1; Supplementary Table S2) and measured the 2-methylcitrate synthase and 2-methylisocitrate lyase activities in the hyphae of the wild type, $\Delta m c s 1$, and $\triangle m c l 1$. Deletion of MCS1 or MCL1 led to greatly decreased 2-methylcitrate synthase or 2-methylisocitrate lyase activities (Fig. 2A and B), implying that MCSI encodes a 2-methylcitrate synthase and MCL1 encodes a 2-methylisocitrate lyase in $P$. oryzae. To determine cellular organelles where the methylcitrate cycle works, we colocalized enhanced green fluorescent protein (GFP)-tagged Mcl1 (GFP-Mcl1) and DsRED2-tagged Atp1 (Atp1-RED), a mitochondrial inner membrane marker protein (Shi et al. 2018), in P. oryzae. The signals of GFP-Mcl1 and Atp1-RED were well overlapped (Fig. 2C), suggesting that Mcl1 is localized in the mitochondria.

\section{MCL1 and MCS1 are involved}

in propionate metabolism in $P$. oryzae.

The $\Delta m c l 1$ mutant was sensitive to a low concentration of propionate $(0.002 \%)$ but the wild type, $\Delta m c s 1$, and the doubledeletion mutant $\Delta m c s 1 \Delta m c l 1$ were not (Fig. 3A). Isoleucine, valine, and methionine are converted into propionyl-CoA; glutamic acid, however, does not produce propionyl-CoA during catabolism. On MM containing $\mathrm{NaNO}_{3}, \Delta m c l 1$ grew slower than the wild type, whereas $\Delta m c s 1$ had growth similar to that of the wild type. Relative to those on medium containing $\mathrm{NaNO}_{3}$ 
A
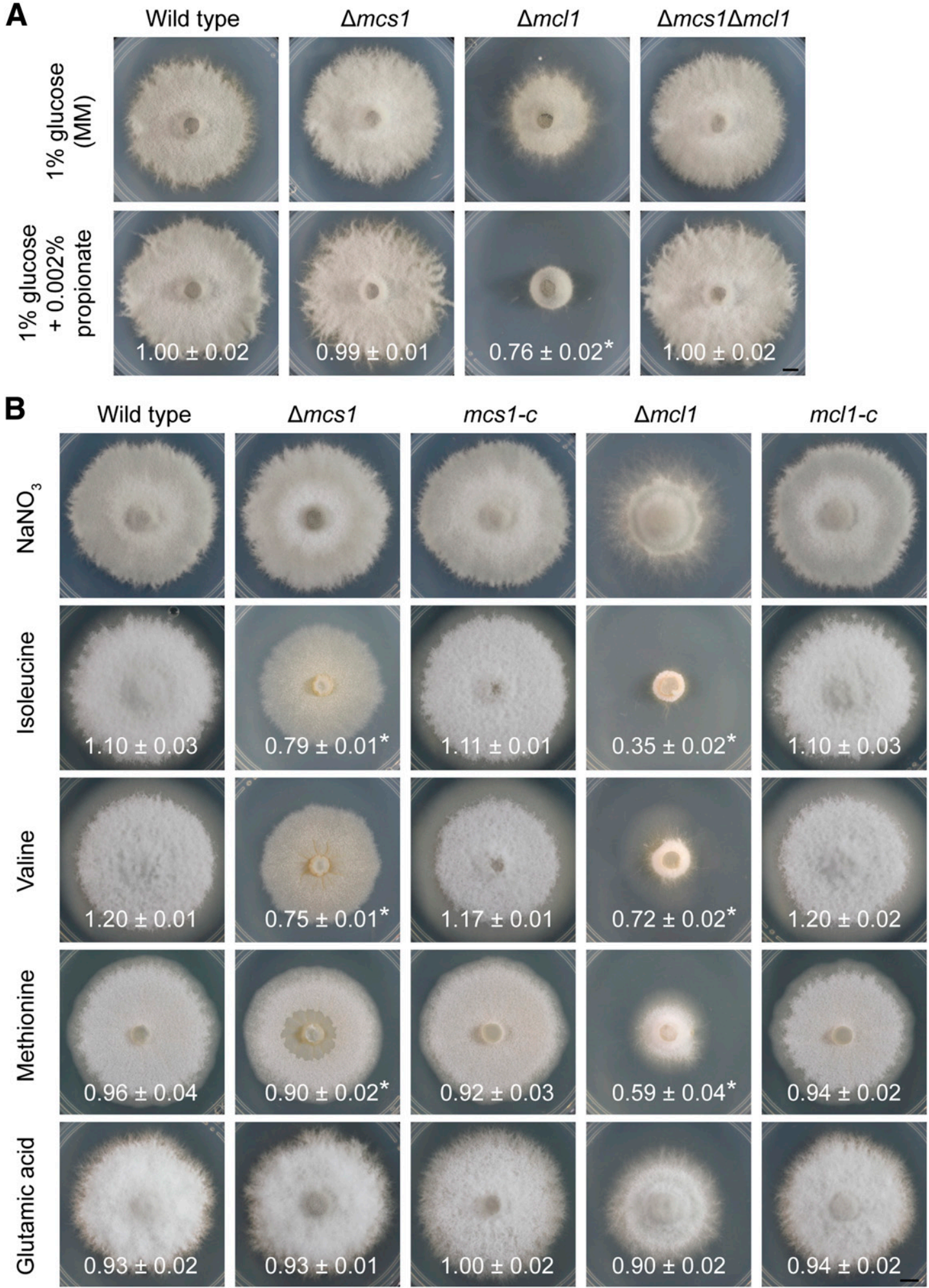

Fig. 3. The role of MCL1 and MCS1 in propionate metabolism. A, A low concentration of propionate inhibits the growth of $\Delta m c l 1$ but not of $\Delta m c s 1$ or $\Delta m c s 1 \Delta m c l 1$. Strains were cultured on minimal medium (MM) (1\% glucose) with or without $0.002 \%$ propionate for 10 days. B, Propionyl-CoA-producing amino acids inhibit the growth of $\Delta m c l 1$ and $\Delta m c s 1$. The wild type, $\Delta m c s 1, \Delta m c l l$, and their complementation strains $m c s 1-c$ and $m c l 1-c$ were cultured on MM $\left(\mathrm{NaNO}_{3}\right)$ and $\mathrm{MM}$ in which $\mathrm{NaNO}_{3}$ was replaced by $25 \mathrm{mM}$ isoleucine, valine, methionine (producing propionyl-CoA), or glutamic acid (without producing propionyl-CoA) as the sole nitrogen source for 10 days. In A and B, numbers (mean \pm standard deviation) are relative growth rate of substrate mycelia in treatment groups, compared with those in MM. Asterisks indicate significant differences between the mutant and the wild type as estimated by Tukey's honestly significant difference test, $P<0.05$. 
or glutamic acid, the $\Delta m c l l$ and $\Delta m c s 1$ mutants, especially $\Delta m c l 1$, grew more slowly and had fewer aerial hyphae than the wild type when using isoleucine, valine, and methionine as the sole nitrogen source (Fig. 3B). The $\Delta m c l 1$ mutant could not grow on $\mathrm{CM}$ with propionate as the sole carbon source, whereas the $\Delta m c s 1$ mutant had a strongly reduced growth rate relative to the wild type on such medium (Fig. 4A). On MM with propionate or the $\mathrm{C} 5$ fatty acid valerate as the sole carbon source, both $\Delta m c l 1$ and $\Delta m c s 1$ could not grow (Fig. 4A). The growth of $\Delta m c l 1$ and $\Delta m c s 1$ was recovered in the complementation strains $m c l 1-c$ and $m c s l-c$ on MM with isoleucine, valine, or methionine as the sole nitrogen source or with propionate as the sole carbon source (Figs. 3B and 4A). The MCS1 and MCL1 expression levels in the hyphae cultured in different carbon sources were examined by quantitative real-time PCR (qRTPCR) (Fig. 4B and C). Compared with glucose (MM), MCS1 and $M C L 1$ transcript levels were significantly upregulated in the media with isoleucine, valine, heptadecanoic acid $\left(\mathrm{C}_{17} \mathrm{H}_{34} \mathrm{O}_{2}\right)$, acetate, or oleic acid $\left(\mathrm{C}_{18} \mathrm{H}_{34} \mathrm{O}_{2}\right)$ but not glutamic acid as the sole carbon source $(P<0.05)$. These results suggested that $M C L 1$ and $M C S 1$ are required for propionate utilization, and deletion of MCS1 in $\Delta m c l 1$ eliminates the sensitivity of $\triangle m c l l$ to a low concentration of propionate, suggesting that MCL1 and MCS1 play diverse roles in fungal growth.

\section{$M C L 1$ and $M C S 1$ are required \\ for growth and conidiation in $P$. oryzae.}

Both $\Delta m c s 1$ and $\Delta m c l 1$ grew more slowly than the wild type on CM (Fig. 5A and B). Mutant $\Delta m c l 1$ produced fewer conidia, approximately one-sixth of the wild type (Fig. 5C). However, on CM, $\Delta m c s 1$ had sparse and short aerial hyphae (Fig. 5A) and did not produce any conidia (Fig. 5C). Mutant $\Delta m c s 1$ had dense aerial hyphae on MM, similar to the wild type (Fig. 3A). Because hydrophobins are necessary for the formation of aerial hyphae (Elliot and Talbot 2004; Shi et al. 2018), we measured the expression level of hydrophobin genes in mycelia of $\Delta m c s 1$ cultured on CM or MM. Compared with the wild type, the transcript levels of three hydrophobin genes (MGG_09134, MGG_10105, and $M P G 1)$ in $\triangle m c s 1$ were downregulated on both CM and MM, whereas MHP1 was upregulated on MM (Fig. 5D). However, $\triangle m c s 1$ had higher transcript levels of four hydrophobin genes (MHP1, MGG_09134, MGG_10105, and MPG1) on MM compared with CM. These results were consistent with the phenotype of aerial mycelia of $\Delta m c s$ l: sparse aerial hyphae on CM and dense but easily collapsed hyphae on MM (Fig. 5A and E). The conidial germination and appressorium formation of $\Delta m c l 1$ is comparable with that of the wild type. However, the conidial germination of $\Delta m c l l$ was easily impaired by propionate addition, relative to the wild type (Fig. $5 \mathrm{~F}$ and $\mathrm{G}$ ). In the presence of $1 \%$ propionate, the spores of $\Delta m c l l$ had lower germination rates and shorter germ tubes than in $\mathrm{H}_{2} \mathrm{O}$ (Fig. 5F and $\mathrm{G}$ ).

\section{MCL1 is involved in virulence of $P$. oryzae.}

Mutant $\Delta m c l 1$ produced conidia on CM but $\Delta m c s 1$ did not; therefore, we assayed the virulence of $\Delta m c l l$ on rice by spraying a conidial suspension onto rice seedlings. Compared with the wild type and the complemented strain mcll-c, the disease severity caused by $\Delta m c l l$ on rice was reduced (Fig. $6 \mathrm{~A}$ and $\mathrm{B}$ ). Nevertheless, $\Delta m c l 1$ still produced a few typical, spindle-like blast lesions with gray centers on rice leaves. We then observed the infection process of $\Delta m c l l$ on barley leaves using droplet assays. At $24 \mathrm{hpi}, 40.0 \%$ of the wild-type appressoria had produced infection hyphae, while no appressoria of $\Delta m c l l$ had formed penetration pegs. At $48 \mathrm{hpi}, 38.2 \%$ of $\Delta m c l 1$ appressoria finished penetration and produced short infection hyphae which were limited to the initially penetrated cells, while the wild-type infection hyphae had completed cross-wall penetration and spread among cells (Fig. 6C and D). The wild type also produced secondary aerial, spore-forming hyphae on lesions earlier than $\Delta m c l l$. An appressorium penetrates the plant cuticle primarily mediated by its huge intracellular turgor pressure in $P$. oryzae (de Jong et al. 1997). The appressorium turgor of $\Delta m c l l$ was evaluated by an incipient cytorrhysis assay (Liu et al. 2007). Compared with the wild type and the complemented strain, more collapsed appressoria were observed in $\Delta m c l 1$ under hyperosmotic glycerol solutions (Fig. 6E), suggesting that $\Delta m c l l$ had lower appressorium turgor than the wild type. The mycelial blocks of $\Delta m c l l$ were inoculated on intact rice leaves and caused weak lesions; however, they caused severe lesions on abraded rice leaves, similar to the wild type (Fig. 7), also suggesting decreased penetration ability of $\Delta m c l 1$.

Icl1, an isocitrate lyase, is homologous to Mcl1 in protein sequence (57\% identity) and is involved in the glyoxylate cycle in P. oryzae (Wang et al. 2003). We deleted ICL1 in the wildtype strain 70-15 and $\Delta m c l 1$, generating $\Delta i c l l$ and the ICLI and $M C L 1$ double deletion mutant $\Delta i c l 1 \Delta m c l 1$. In virulence assays, $\Delta i c l 1$ displayed similar virulence to $\Delta m c l l$, and its virulence was weaker than the wild type. Mutant $\Delta i c l 1 \Delta m c l 1$, however, had much weaker virulence than both $\Delta i c l l$ and $\Delta m c l l$, and only caused pinpoint-like brown specks on rice leaves (Fig. 6A), implying that Icl1 and Mcl1 have a synergistic effect in the infection process of $P$. oryzae. Because Icl1 is essential for lipid utilization (Wang et al. 2003), we tested the growth of $\Delta i c l 1 \Delta m c l l$ on the media using olive oil or glucose as the sole carbon source. On MM without glucose (MM-C) supplemented with $1 \%$ olive oil as the sole carbon source, $\Delta i c l 1 \Delta m c l 1$ grew very slowly, similar to $\Delta i c l l$, and could not utilize lipids efficiently, whereas $\Delta m c l l$ grew similarly to the wild type. On MM containing glucose, $\Delta i c l 1 \Delta m c l 1$ grew similarly to $\Delta m c l l$ and slower than the wild type, whereas $\Delta i c l l$ grew similarly to the wild type (Fig. 8A). On CM, $\Delta i c l 1 \Delta m c l 1$ grew similarly to $\Delta m c l l$ and slower than the wild type and $\Delta i c l l$. On CM without glucose (CM-C) supplemented with $1 \%$ olive oil, $\Delta i c l 1 \Delta m c l 1$ produced fewer aerial mycelia than $\Delta i c l 1, \Delta m c l 1$, and the wild type (Fig. 8B). In liquid media, however, $\Delta i c l 1 \Delta m c l 1$ did not show significant differences in growth from $\Delta i c l 1$ and $\Delta m c l l$ (Fig. 8C). These results suggested that Icl1 is essential for lipid utilization, whereas Mcl1 is likely involved in utilization of glucose during fungal growth.

\section{Acetate relieves the toxicity of propionate.}

On MM, $0.08 \%$ sodium propionate hindered the growth of the wild type, $\Delta m c s 1$, and $\Delta m c l 1$; addition of sodium acetate recovered the growth of tested strains, especially the wild type and $\Delta m c s 1$, which was severely inhibited by propionate (Fig. 9A). The growth recovery was dependent on the acetate concentrations. One molecule each of acetyl-CoA and propionylCoA were produced from valerate after $\beta$-oxidation. Valerate, however, inhibited the growth of the wild type, $\Delta m c s 1$, and $\Delta m c l 1$ (Fig. 9B). On CM, acetate also recovered conidiation, which was defective in $\Delta m c s 1$ and $\Delta m c l l$ (Fig. 9B). We used the conidia produced by $\Delta m c s 1$ on $\mathrm{CM}$ after adding $0.10 \%$ sodium acetate to test its conidial germination rate, appressorium formation rate, and virulence by droplet assays. The conidial germination and appressorium formation rate of $\Delta m c s 1$ was comparable with that of the wild type. The conidia of $\Delta m c s 1$ caused disease lesions on barley leaves that were similar to those caused by the wild type (Fig. 9C).

\section{$M C S 1$ affects the $\mathrm{NAD}^{+} / \mathrm{NADH}$ ratio and NO production in $P$. oryzae.}

Cellular redox state and NO signaling affect the differentiation of aerial hyphae and conidia (Shi et al. 2018). Because 


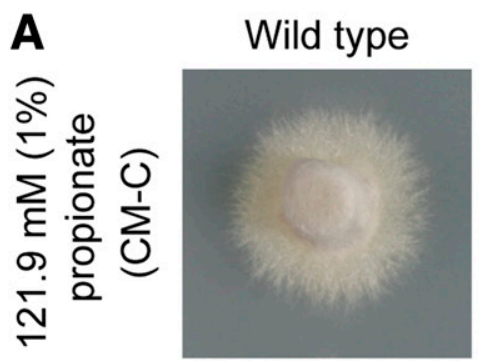

$\Delta m c s 1$
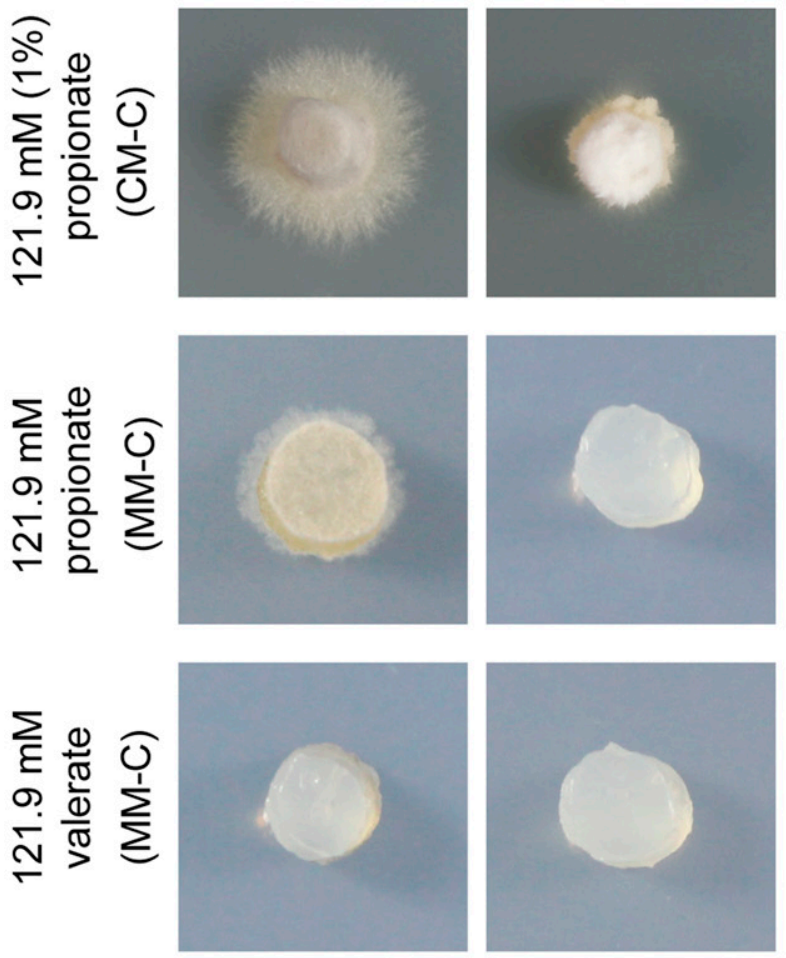

\section{B}

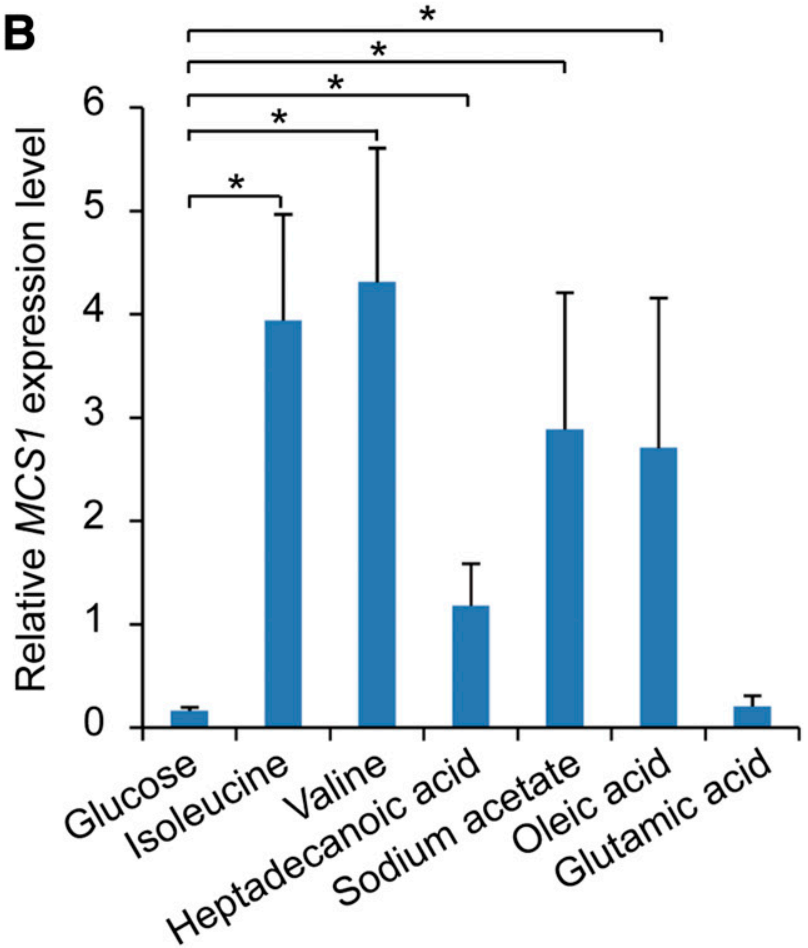

$\operatorname{mcs} 1-c$
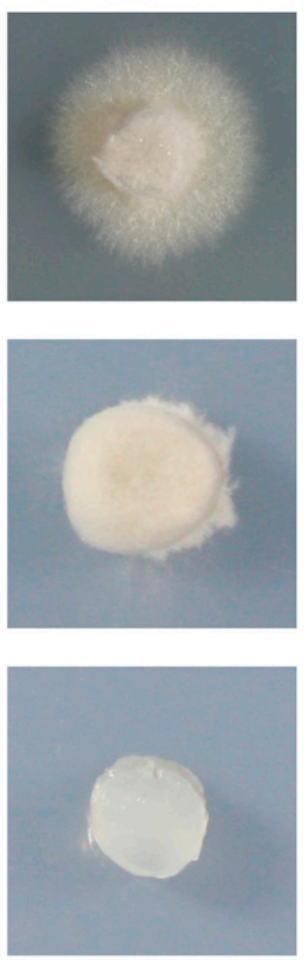

C

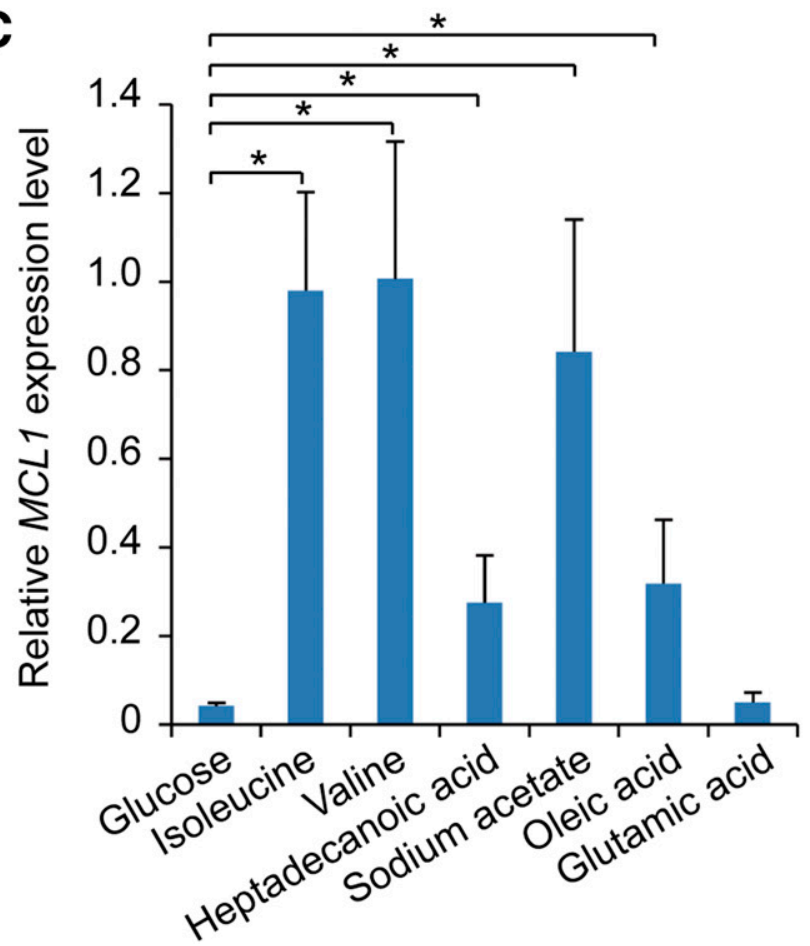

Fig. 4. MCS1 and MCL1 are required for propionate utilization. A, Growth of the wild type, $\Delta m c l 1, \Delta m c s 1$, and their complementation strains $m c s 1$ - $c$ and $m c l 1$ $c$ on the media with propionyl-CoA-producing carbon sources. Strains were cultured on complete medium (CM) in which $1 \%$ glucose was replaced by $1 \%$ $(=121.9 \mathrm{mM})$ propionate for 7 days $(\mathrm{CM}-\mathrm{C})$, and on minimal medium $(\mathrm{MM})$ in which $1 \%$ glucose was replaced by $121.9 \mathrm{mM}$ propionate or valerate (producing propionyl-CoA and acetyl-CoA) as the sole carbon source for 25 days (MM-C). Bar = $5 \mathrm{~mm}$. B and C, Relative expression levels of $M C S 1$ and $M C L 1$ in the wild-type hyphae cultured in MM (glucose) and MM in which glucose was replaced by $25 \mathrm{mM}$ isoleucine, $25 \mathrm{mM}$ valine, $1 \%$ heptadecanoic acid (producing propionyl-CoA), $1 \%$ sodium acetate, $0.01 \%$ oleic acid (producing acetyl-CoA), or $25 \mathrm{mM}$ glutamic acid (without producing propionyl-CoA and acetyl-CoA) as the sole carbon source. $\beta$-TUBULIN and $\alpha$-ACTIN were used as reference genes. Asterisks indicate significant differences between two columns as estimated by Tukey's honestly significant difference test, $P<0.05$. 
A

Wild type

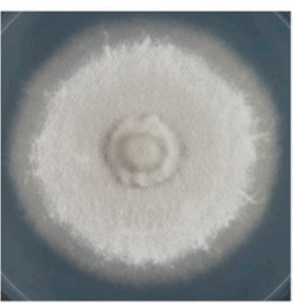

$\mathrm{CM}$

CM
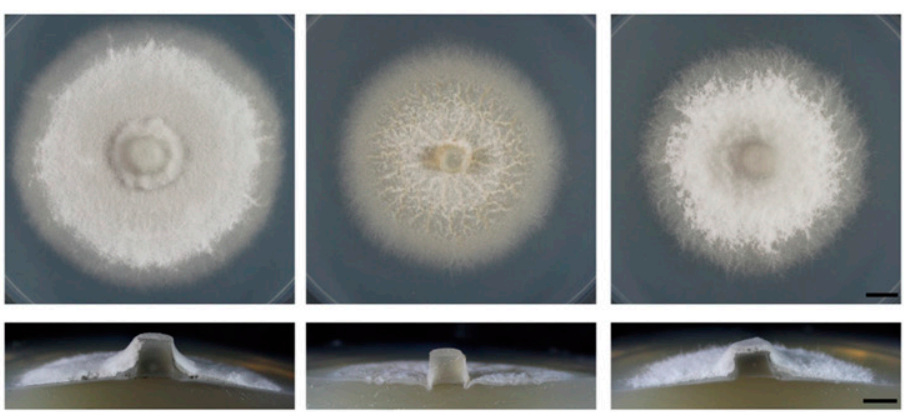

C

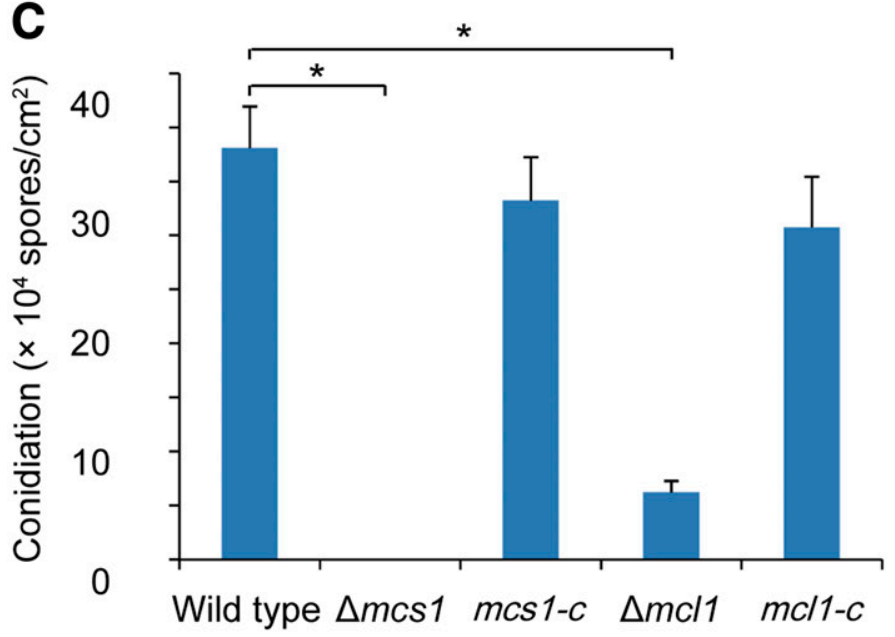

D

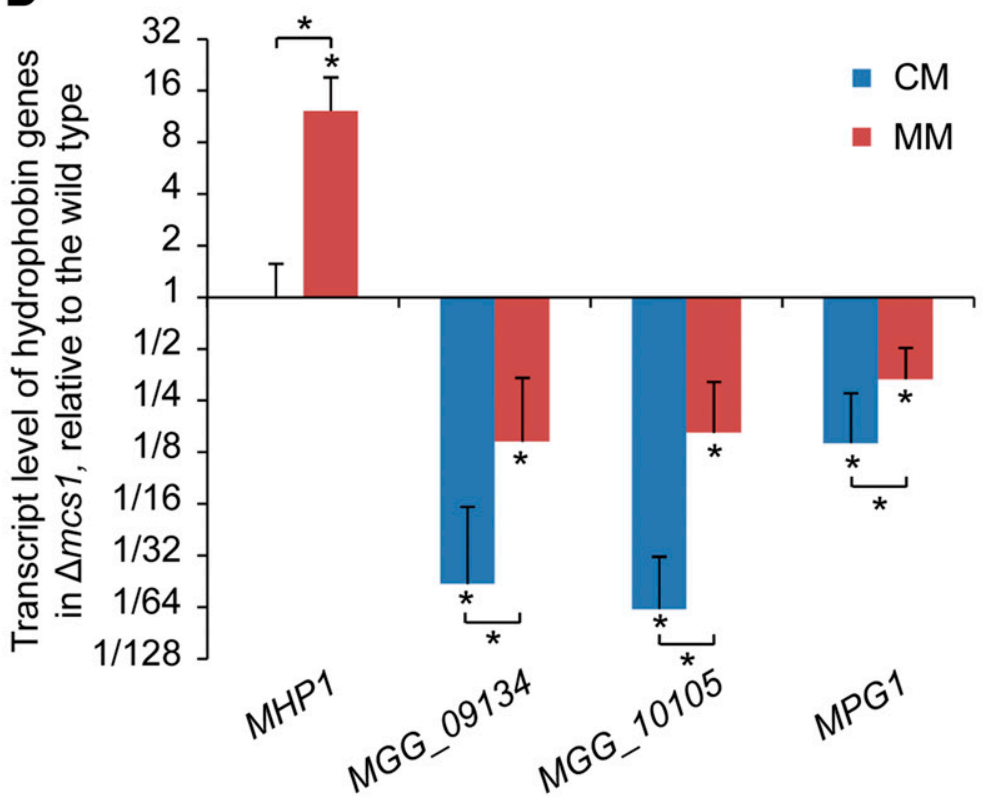

B
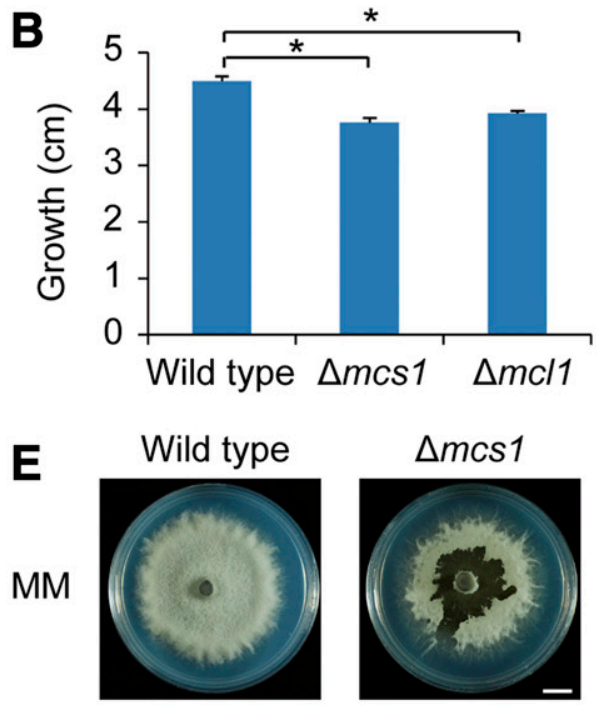

$\mathbf{F}$

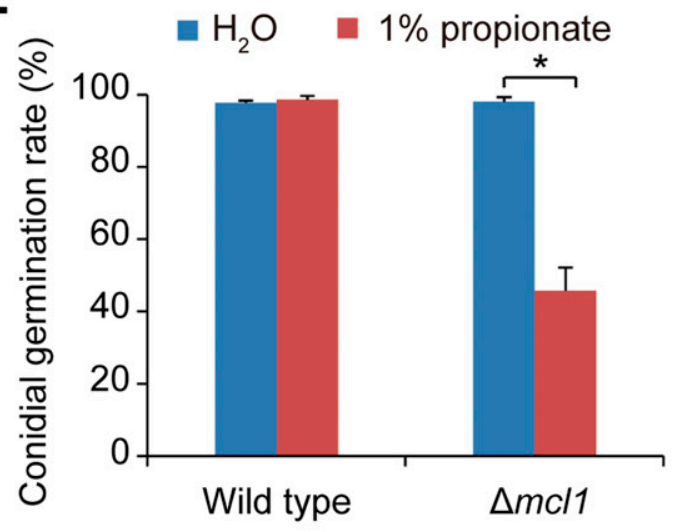

G

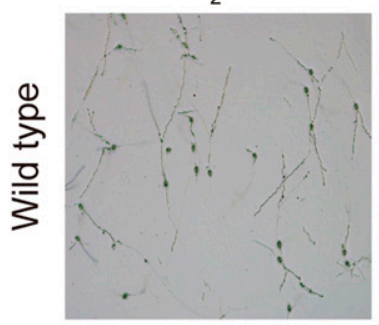

$1 \%$ propionate

Fig. 5. Phenotypic assays of $\Delta m c s 1$ and $\Delta m c l 1$. A, Colony morphology of $\Delta m c s 1$ and $\Delta m c l 1$. Strains were cultured on complete medium $(\mathrm{CM})$ for 8 days. Bar $=$ $5 \mathrm{~mm}$. B, Growth of $\Delta m c s 1$ and $\Delta m c l 1$. The diameter of mycelial colonies on CM was measured after 8 days. C, Conidiation of the wild type, $\Delta m c s 1, \Delta m c l 1$, and their complemented strains $m c s 1-c$ and $m c l 1-c$ cultured on CM for 8 days. D, Relative transcript level of hydrophobin genes in the aerial mycelia of $\Delta m c s 1$ cultured on CM or minimal medium (MM) compared with the wild type. $\beta$-TUBULIN and $\alpha$-ACTIN were used as reference genes in quantitative real-time PCR. E, Collapsed aerial mycelia of $\Delta m c s 1$ on MM incubated at $25^{\circ} \mathrm{C}$ under a cycle of $16 \mathrm{~h}$ of light and $8 \mathrm{~h}$ of darkness for $12 \mathrm{days}$. Bar $=10 \mathrm{~mm}$. F, Conidial germination of $\Delta m c l l$ was impaired by propionate. Germination of spores in $\mathrm{H}_{2} \mathrm{O}$ or $1 \%$ propionate was counted at $24 \mathrm{~h}$ postinoculation (hpi). G, Morphology of germinated spores in propionate at $24 \mathrm{hpi}$. Bar $=20 \mu \mathrm{m}$. Asterisks indicate significant differences between columns in figures or between the mutant and the wild type as estimated by Tukey's honestly significant difference test $(P<0.05)$. Error bars represent standard deviation. 

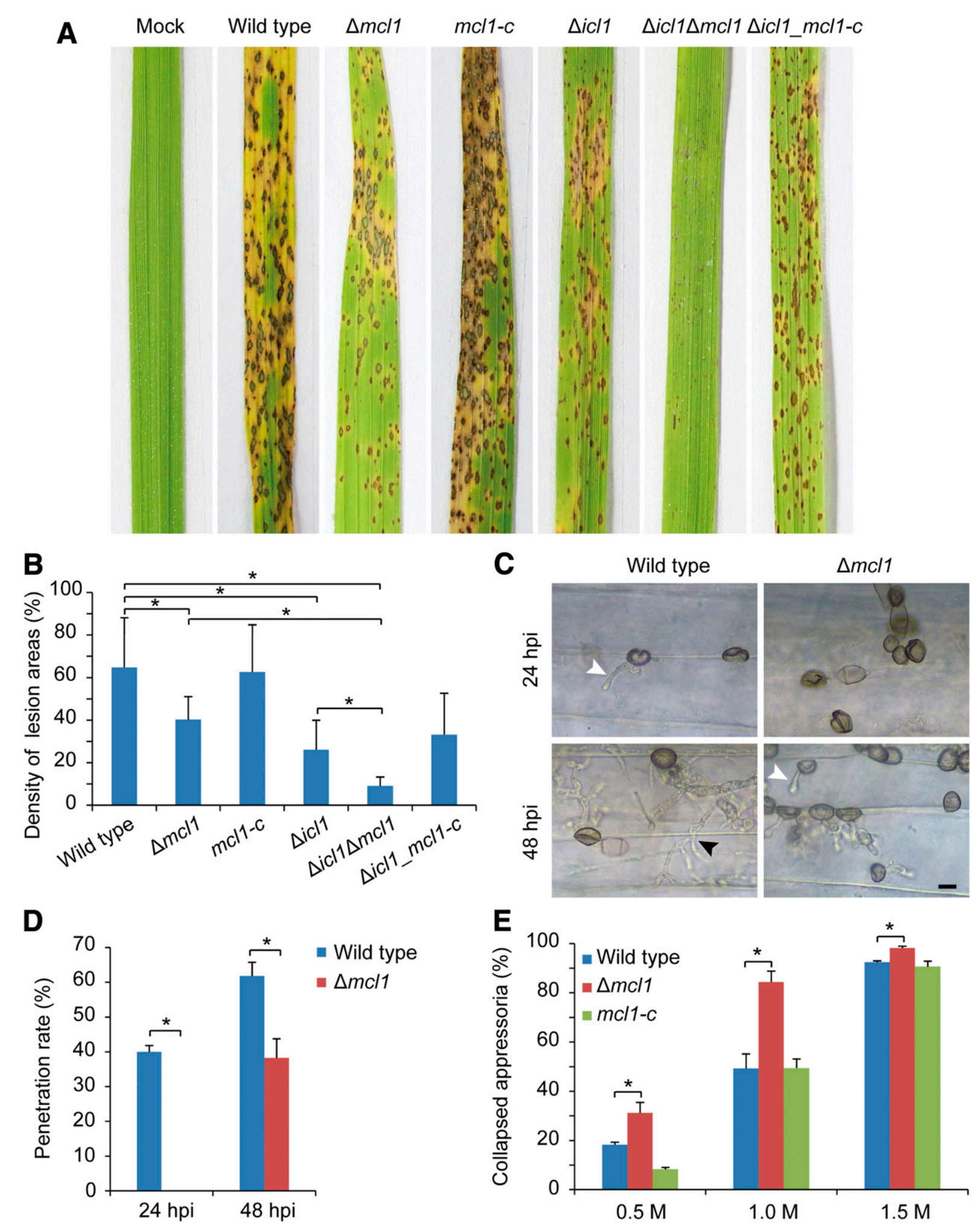

Fig. 6. Virulence assays of $\Delta m c l 1$. A, Virulence tests. The conidial suspension $\left(1 \times 10^{5}\right.$ spores $\left.\mathrm{ml}^{-1}\right)$ of the wild type, $\Delta m c l 1, \Delta i c l 1, \Delta i c l 1 \Delta m c l 1, m c l 1-c(M C L 1$ was complemented in $\Delta m c l 1)$, and $\Delta i c l 1 \_m c l 1-c$ ( $M C L 1$ was complemented in $\left.\Delta i c l 1 \Delta m c l 1\right)$ was sprayed on rice seedlings and incubated for 7 days. B, Disease lesion severity assays for tests in A. C, Images of invasive hyphae of the wild type and $\Delta m c l l$ on barley epidermal cells. White arrows = invasive hyphae and black arrow $=$ invasive hyphae which penetrated into the second cell. Bar $=10 \mu \mathrm{M}$. D, Penetration rate by appressoria of the wild type and $\Delta m c l 1$ on barley cuticle. E, Assessment of appressorium turgor pressure by incipient cytorrhysis assay. Appressoria at $48 \mathrm{~h}$ postinoculation were immersed in glycerol solutions $(0.5,1.0$, and $1.5 \mathrm{M})$, and the collapsed appressoria were counted. Error bars represent standard deviation. Asterisks indicate significant differences as estimated by Tukey's honestly significant difference test $(P<0.05)$. 
$\Delta m c s 1$ grown on CM produced sparse and short aerial hyphae and failed to differentiate into conidia, we measured the contents of NAD and NO in the mycelia of $\Delta m c s 1$ cultured on CM under a light-dark cycle. Compared with the wild type, $\Delta m c s 1$ had a lower $\mathrm{NAD}^{+} / \mathrm{NADH}$ ratio (Fig. 10A), suggesting that the hyphal cells of $\Delta m c s 1$ have stronger reducing power. Mutant $\Delta m c s 1$ had an NO content of $1.61 \pm 0.25 \mu \mathrm{mol} \mathrm{g}$ protein $^{-1}$, much lower than that in the wild type (Fig. 10B).

\section{DISCUSSION}

In fungi, several amino acids (valine, isoleucine, methionine, and threonine), odd-chain fatty acids, and propionate are metabolized via the methylcitrate cycle. In this study, we found the homologous genes encoding the five enzymes involved in the methylcitrate cycle in $P$. oryzae, and functionally identified Mcs1 and Mcl1 as a 2-methylcitrate synthase and a 2-methylisocitrate lyase, respectively, by genetic and phenotypic assays. Furthermore, we evaluated the roles of the methylcitrate cycle in fungal development and virulence of P. oryzae.

The methylcitrate cycle is required for fungal growth, conidiation, appressorium turgor, plant penetration, virulence, cellular redox state, and NO production (Figs. 1A and 10) by enabling detoxification of propionyl-CoA and 2-methylisocitrate in P. oryzae. Deletion of MCS1 led to slowed mycelial growth, deficient aerial hyphal differentiation, and blocked conidiation in P. oryzae (Fig. 5). Deletion of MCL1 led to slowed mycelial growth, decreased conidiation, and reduced virulence (Figs. 5 and 6). Hydrophobin proteins, cellular redox state $\left(\mathrm{NAD}^{+} / \mathrm{NADH}\right.$ ratio), and $\mathrm{NO}$ signaling play important roles in aerial hyphae differentiation and conidiation in fungi (Shi et al. 2018; Zhang et al. 2015). Deficiencies in aerial hyphal differentiation and conidiation in $\Delta m c s l$ were partly caused by downregulation of hydrophobin genes, lowered $\mathrm{NAD}^{+} / \mathrm{NADH}$ ratio, and decreased NO content in the mycelia of P. oryzae (Figs. 5D and 10). In humans, 2-methylcitrate affects the TCA cycle by inhibiting citrate synthase, aconitase, $\mathrm{NAD}^{+}$- and $\mathrm{NADP}^{+}$-linked isocitrate dehydrogenase, phosphofructokinase, and the tricarboxylase carrier (CheemaDhadli et al. 1975). In the yeast Yarrowia lipolytica, the methylcitrate cycle is important for glycerol metabolism

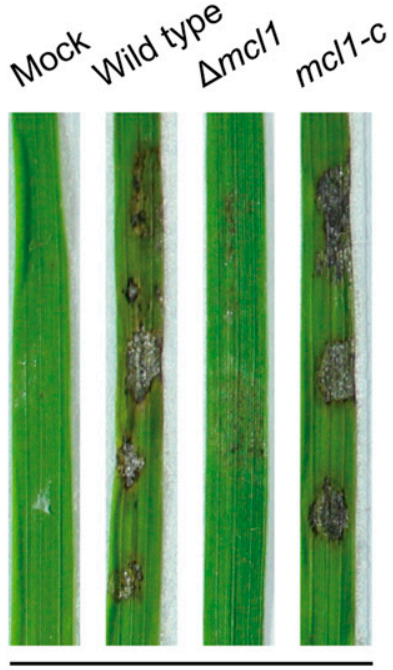

Intact

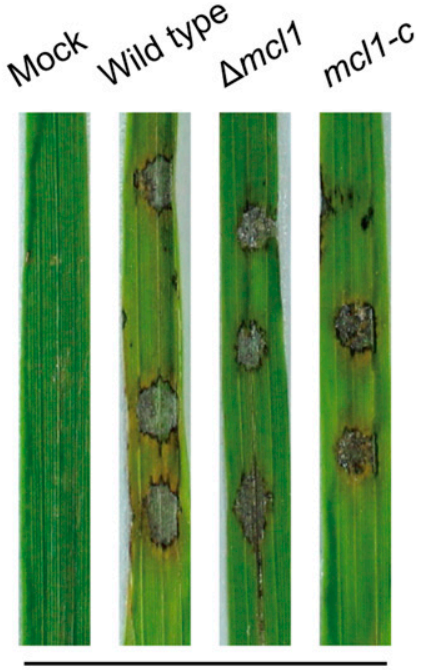

Abraded
Fig. 7. Mycelial virulence of $\Delta m c l 1$ on abraded rice leaves. Mycelial blocks were inoculated onto intact and abraded rice leaves for 4 days.
(Papanikolaou et al. 2013). Produced by a high concentration of glycerol, the appressorium turgor is a main force for $P$. oryzae to penetrate the plant cuticle and initiate infection (de Jong et al. 1997). The reduced virulence of $\Delta m c l l$ could be due to its lowered appressorium turgor and slowed cuticle penetration (Fig. 6C, D, and E). Compared with intact rice leaves, the recovered virulence of $\Delta m c l l$ on abraded rice leaves also suggested that the deficient virulence of the mutant was partly caused by its impaired penetration ability (Fig. 7).

The metabolic reactions catalyzed by Mcs1 and Mcl1 are the first and last step, respectively, in the conversion from propionyl-CoA to pyruvate (Fig. 1). Although both Mcs1 and Mcl1 are required for propionyl-CoA metabolism and fungal development, the phenotypic differences in development and virulence between $\Delta m c s 1$ and $\Delta m c l l$ were obvious. Mutant $\Delta m c s 1$ displayed more severe defects in aerial hyphal differentiation and conidiation than $\Delta m c l 1$ (Fig. 5A and C). The conidia of $\Delta m c l l$ showed reduced virulence, while $\Delta m c s 1$ did not (Figs. 6A and 9C). Differences were also found between $\Delta m c s 1$ and $\Delta m c l 1$ in the utilization of propionate as the sole carbon source (Fig. 4A) or isoleucine, valine, methionine, and threonine as the sole nitrogen source (Fig. 3B; Supplementary Fig. S2), as well as in the resistance to the growth inhibition caused by propionate in the presence of glucose (Figs. 3A and 9A). Mutant $\Delta m c l l$ was more sensitive than $\Delta m c s l$ to propionate, isoleucine, and methionine during growth (Fig. 3A and B). The phenotypic differences between $\Delta m c s 1$ and $\Delta m c l 1$ are likely caused by a discrepancy in the accumulation of different types of intermediate metabolites. The toxicity of different metabolites to different target proteins varies. In A. fumigates and $A$. nidulans, propionyl-CoA accumulated in $\triangle m c s A$, a 2methylcitrate synthase deletion mutant (Ibrahim-Granet et al. 2008; Zhang et al. 2004). In A. nidulans, 2-methylisocitrate was secreted to the medium by $\Delta m c l A$, a 2-methylisocitrate lyase deletion mutant, but not by the wild type when cultured with propionate. This methylisocitrate excretion, however, was competitively inhibited by acetate (Brock 2005). In $\Delta m c l 1$, the metabolites methylcitrate and 2-methyl-cis-aconitate are also likely accumulated in cells (Fig. 1A). In A. nidulans, propionyl$\mathrm{CoA}$ inhibits the activity of CoA-dependent enzymes (such as pyruvate dehydrogenase, succinyl-CoA synthetase, and ATPcitrate lyase) and interferes with the balance of carbon metabolism (Brock and Buckel 2004), while 2-methylisocitrate competitively inhibits the activity of the NADP-dependent isocitrate dehydrogenase (Brock 2005). In humans, propionylCoA uncompetitively inhibits the pyruvate dehydrogenase complex (Schwab et al. 2006), while 2-methylcitrate inhibits citrate synthase, aconitase, and the $\mathrm{NAD}^{+}$- and $\mathrm{NADP}^{+}$-linked isocitrate dehydrogenase (Cheema-Dhadli et al. 1975). Propionyl-CoA, 2-methylisocitrate, and 2-methylisocitrate seem to function on different enzymes and have different physiological roles, leading to diverse and distinct phenotypes between mutants $\Delta m c s 1$ and $\Delta m c l 1$. In $P$. oryzae, $\Delta m c s 1 \Delta m c l 1$ showed sensitivity to $0.002 \%$ propionate similar to that of $\Delta m c s 1$ but lower sensitivity than $\Delta m c l 1$ (Fig. 3A), implying that 2-methylisocitrate possibly has more severe cytotoxicity in $\Delta m c l l$ than propionyl-CoA in $\Delta m c s 1$ and $\Delta m c s 1 \Delta m c l 1$. On MM containing $0.08 \%$ propionate, $0.10 \%$ acetate completely eliminated the toxicity of propionate on the wild type and $\Delta m c s 1$ but not on $\Delta m c l 1$ (Fig. 9A). In A. fumigatus and A. nidulans, acetate also relieved the growth inhibition of propionate on medium containing glucose (Brock et al. 2000; Maerker et al. 2005). Acetate detoxifies propionyl-CoA by competitively binding CoA to form acetyl-CoA and thereby decreases the production of propionyl-CoA (Fleck and Brock 2008; Limenitakis et al. 2013). Lesser relief on the growth of $\Delta m c l l$ by adding acetate may be due to the accumulation of 
2-methylisocitrate, which could not be substantially removed by acetate in $P$. oryzae.

Fungal 2-methylisocitrate lyases evolved from an ancient isocitrate lyase of the basidiomycete lineage by gene duplication
(Müller et al. 2011). The deletion of ICL1, an isocitrate lyase gene in the glyoxylate cycle, in the $P$. oryzae wild-type strain $70-15$ or Guy11 led to reduced virulence and defects in lipid utilization (Fig. 6A and B) (Wang et al. 2003). Similar to $\Delta i c l 1$,
A
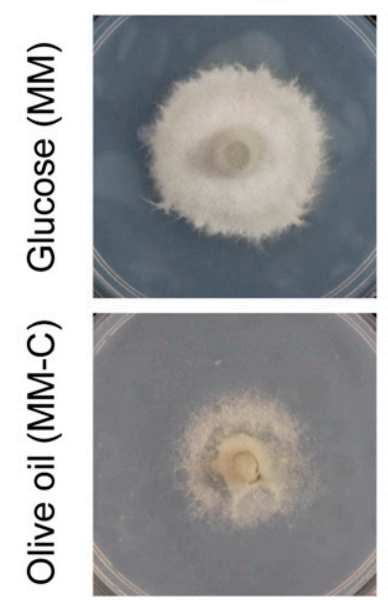

B
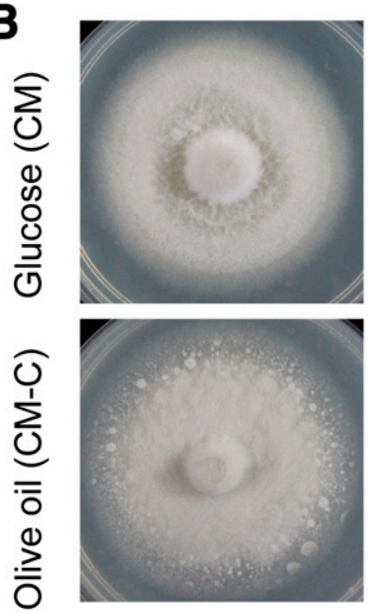

$\Delta i c / 1$
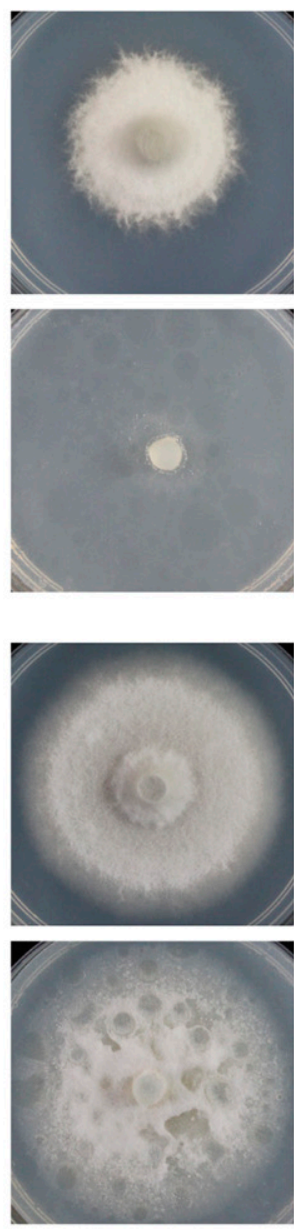

$\Delta m c / 1$
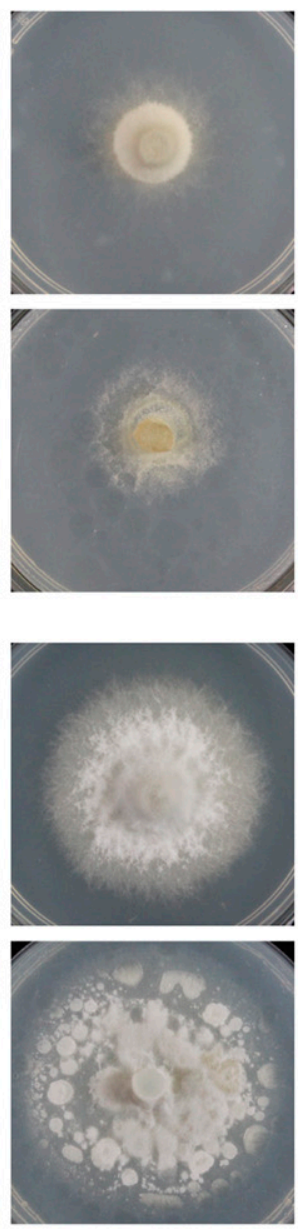

\section{$\Delta i c / 1 \Delta m c l 1$}
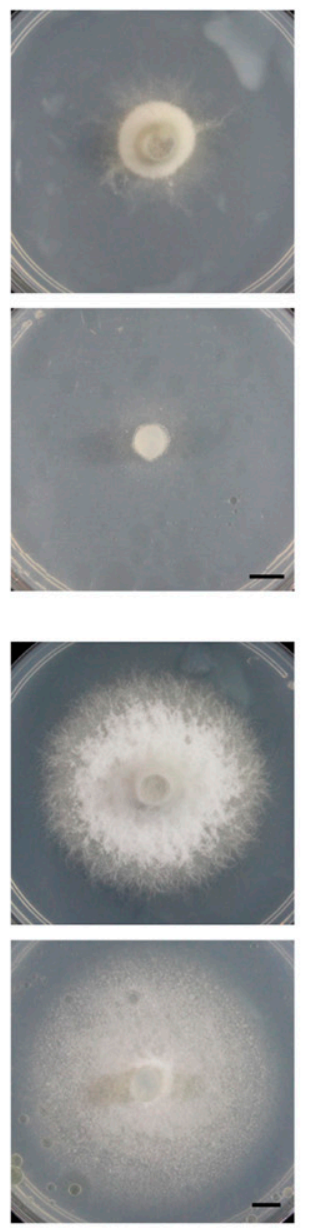

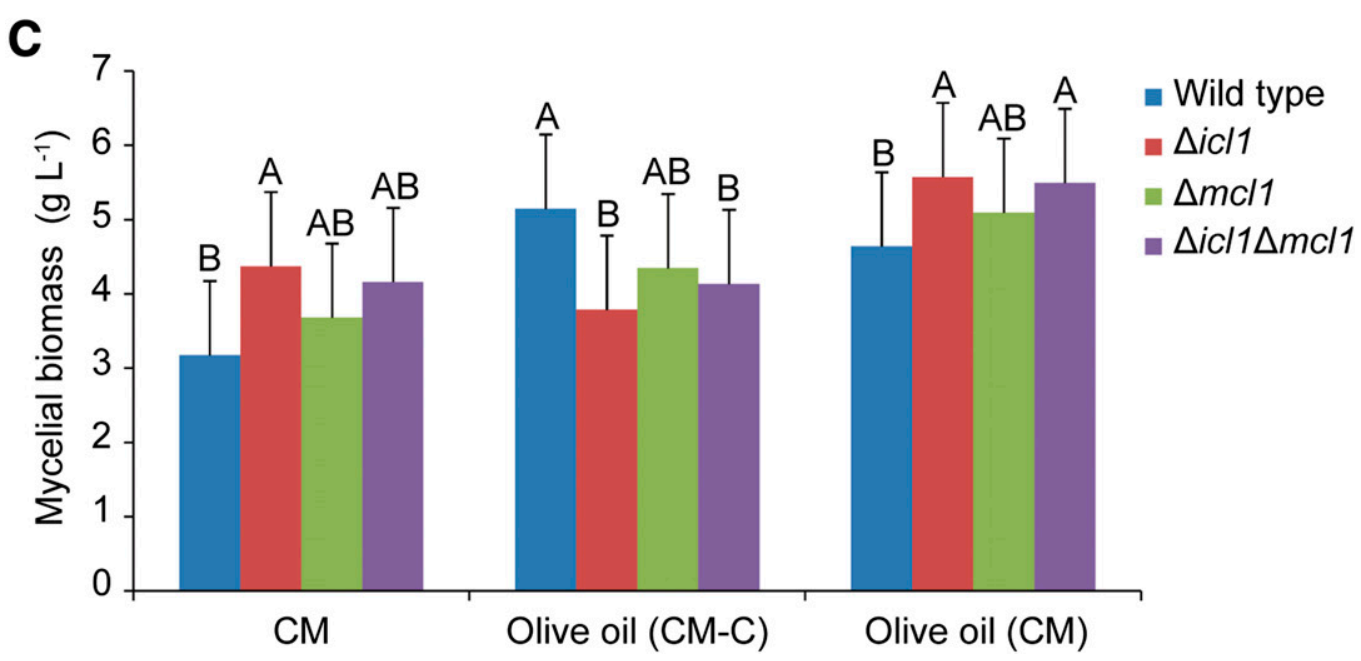

Fig. 8. Role of Icl1 and Mcl1 in utilization of lipids and glucose with and without amino acids present. A, Strains (wild type, $\Delta i c l 1, \Delta m c l 1$, and $\Delta i c l 1 \Delta m c l 1)$ were cultured on minimal medium (MM) and MM without glucose (MM-C) containing $1 \%$ olive oil for 10 days. B, Strains were cultured on complete medium $(\mathrm{CM})$ and $\mathrm{CM}$ without glucose (CM-C) containing $1 \%$ olive oil for 7 days. Bar $=5 \mathrm{~mm}$. C, Mycelial biomass (wet weight) of the strains cultured in liquid CM, $\mathrm{CM}$, or CM-C containing $1 \%$ olive oil for $24 \mathrm{~h}$. Error bars represent standard deviation. Different capital letters indicate significant differences between the strains in the same medium as estimated by Tukey's honestly significant difference test $(P<0.01)$. 
$\Delta m c l 1$ also displayed reduced virulence (Fig. 6A and B). After both ICLI and MCL1 were deleted, the $\triangle i c l 1 \triangle m c l 1$ mutant nearly completely lost its virulence in rice (Fig. 6A). Icl1 and Mcll appear to have a synergistic effect on the virulence of
P. oryzae. In $G$. zeae, both $\Delta i c l 1$ and $\Delta m c l l$ did not show defects in virulence but $\Delta i c l 1 \Delta m c l l$ did, indicating that the impairment of either the glyoxylate cycle or the methylcitrate cycle can be compensated by each other but, in the defects,
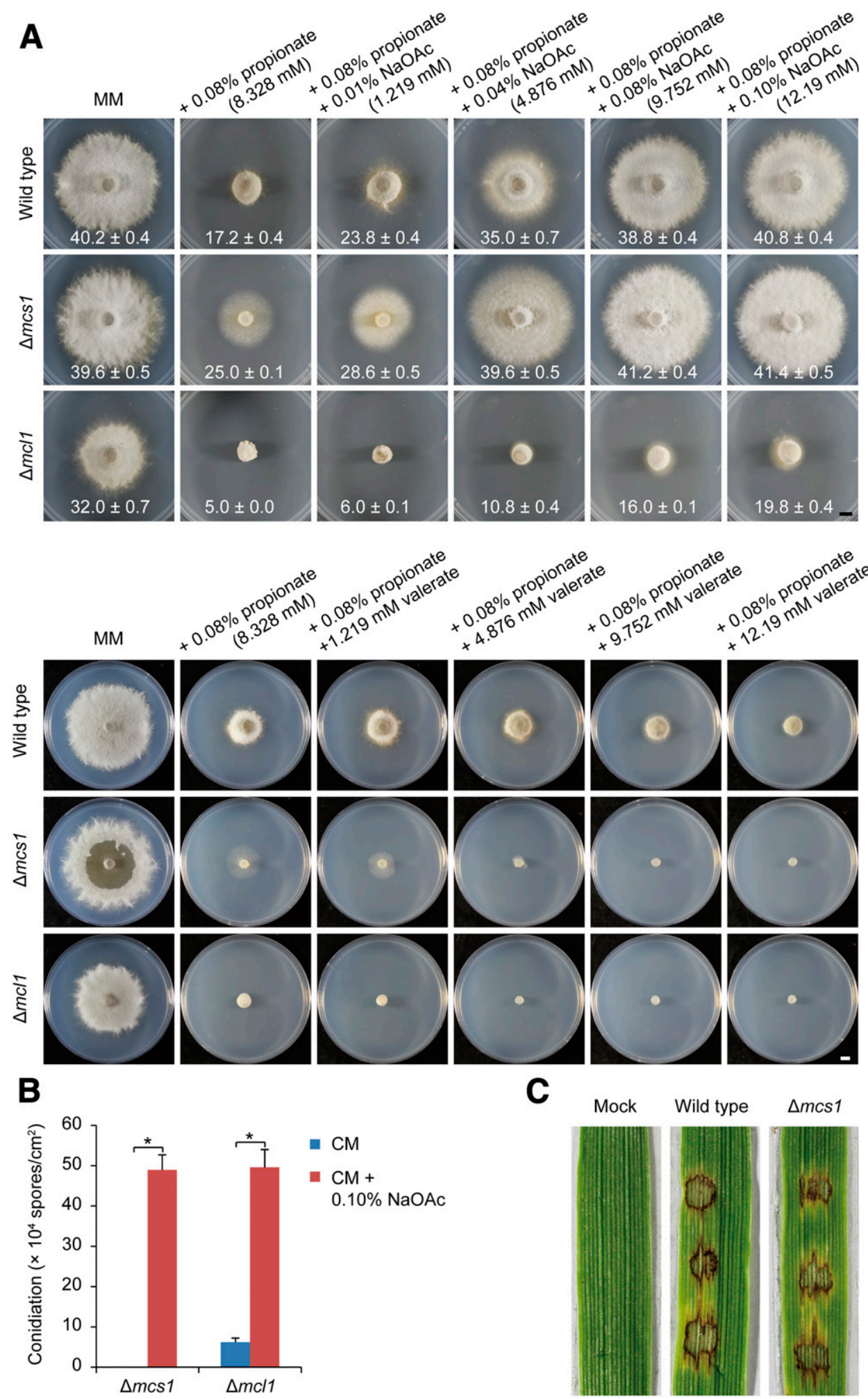

Fig. 9. Inhibition of propionate on fungal growth and conidiation relieved by acetate. A, Colonies of the wild type, $\Delta m c s 1$, and $\Delta m c l 1$ grown on minimal medium (MM) containing $0.08 \%$ sodium propionate and different concentrations of sodium acetate (NaOAc) for 10 days (upper) or sodium valerate for 13 days (lower). Numbers (mean \pm standard deviation $[\mathrm{SD}]$ ) are diameters (in millimeters) of colonies. Bar $=5 \mathrm{~mm}$. B, Sodium acetate recovered conidiation on complete medium (CM); conidiation on $\mathrm{CM}$ was defective in $\Delta m c s 1$ and $\Delta m c l 1$. Error bars represent SD. Asterisks indicate significant differences as estimated by Tukey's honestly significant difference test $(P<0.05)$. C, Virulence tests of $\Delta m c s 1$. Conidial suspension $\left(20 \mu l ; 1 \times 10^{5}\right.$ spores ml $\left.{ }^{-1}\right)$ was dropped onto barley leaves and incubated for 4 days. 
cannot be compensated if both pathways are impaired (Lee et al. 2009). In $P$. oryzae, the greatly reduced virulence in $\Delta i c l 1 \Delta m c l 1$ possibly occurred because $\Delta i c l 1 \Delta m c l 1$ combined both defects of $\Delta i c l l$ and $\Delta m c l l$ in utilizing different carbon sources: $\Delta i c l 1$ displayed defects in utilizing lipids and $\Delta m c l l$ displayed defects in utilizing glucose and amino acids (valine, isoleucine, and methionine) (Figs. 8A and 3B). In Saccharomyces cerevisiae, 2-methylisocitrate lyase is located in the mitochondrial matrix (Luttik et al. 2000). In Y. zipolytica, three enzymes (2-methylcitrate synthase, 2-methylcitrate dehydratase, and 2-methylisocitrate dehydratase) are localized in the mitochondria; 2-methylisocitrate lyase, however, is located in both the mitochondria and cytoplasm (Uchiyama et al. 1982). In Toxoplasma gondii, a one-celled eukaryotic parasite, the 2methylcitrate cycle is split between the mitochondria and cytoplasm, in which 2-methylcitrate synthase is localized in the mitochondria, 2-methylcitrate dehydratase is in the cytoplasm, and 2-methylisocitrate lyase is in the proximity of the mitochondria but in the cytoplasm (Limenitakis et al. 2013). In $P$. oryzae, 2-methylisocitrate lyase (Mcl1) is localized in the mitochondria (Fig. 2C). Disruption of the 2-methylcitrate cycle and the glyoxylate cycle impairs nutrient utilization coupling with various cellular metabolic pathways in the mitochondria and peroxisome (lipid breakdown, glycogen catabolism, and TCA) during fungal growth and appressorial formation. The expression levels of MCL1 and MCS1 were increased not only
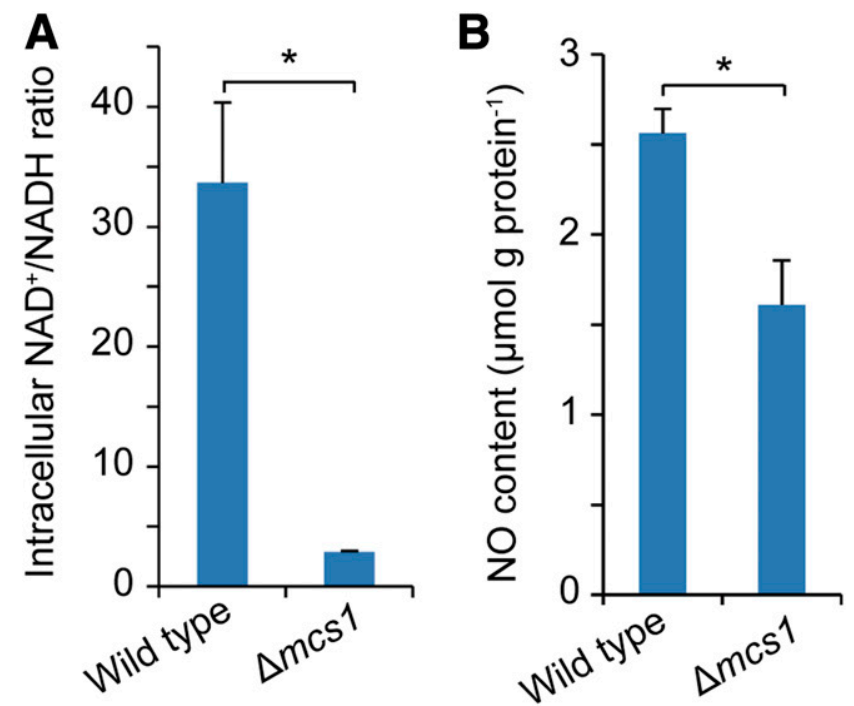

Fig. 10. $\mathrm{NAD}^{+} / \mathrm{NADH}$ ratio and nitric oxide (NO) content in mycelia grown on complete medium $(\mathrm{CM})$. $\mathbf{A}$, Intracellular $\mathrm{NAD}^{+} / \mathrm{NADH}$ ratio in the aerial mycelia of the wild type and $\Delta m c s 1$. B, NO content in the aerial mycelia of the wild type and $\Delta m c s 1$. Error bars represent standard deviation. Asterisks indicate significant differences as estimated by Tukey's honestly significant difference test $(P<0.05)$. by propionyl-CoA-producing carbon sources but also by acetylCoA-producing carbon sources (Fig. 4A and B), suggesting the metabolic connections between the methylcitrate cycle and lipid breakdown, the glyoxylate cycle, or TCA cycles. Therefore, the defects of $\Delta i c l 1 \Delta m c l l$ in metabolizing several carbon sources and amino acids possibly led to a large decrease in virulence.

In summary, we demonstrated that deletion of MCS1 and $M C L 1$ resulted in slowed growth on several carbon or nitrogen sources (propionate, glucose, valine, isoleucine, methionine, and threonine), decreased conidiation, reduced appressorium turgor, and severely delayed appressorial penetration and virulence by interfering with carbon metabolism, cellular redox state $\left(\mathrm{NAD}^{+} / \mathrm{NADH}\right)$, and $\mathrm{NO}$ signaling in $P$. oryzae. Our findings show that the methylcitrate cycle is essential for fungal development and virulence through detoxification of propionyl-CoA, methylcitrate, and 2-methylisocitrate, and plays an important role in maintaining carbon metabolism in P. oryzae.

\section{MATERIALS AND METHODS}

\section{Fungal strains, media, culture conditions, PCR, and statistical tests.}

$P$. oryzae wild-type strain $70-15$ and its derivatives used in this study are listed in Table 1 . Growth tests were performed on CM (Talbot et al. 1993); MM (CM without peptone140, casamino acid, and yeast extract); CM-C; MM-C; MM without $\mathrm{NaNO}_{3}$ (MM-N); MM-C and MM-N supplemented with $25 \mathrm{mM}$ isoleucine, valine, methionine, or glutamic acid; CM-C and MM-C supplemented with $1 \%$ (vol/vol or wt/vol) olive oil, sodium propionate, sodium acetate, heptadecanoic acid, oleic acid, or $121.9 \mathrm{mM}$ sodium valerate; and CM and MM supplemented with different concentrations of sodium propionate, sodium acetate, or sodium valerate at $25^{\circ} \mathrm{C}$ under a cycle of $16 \mathrm{~h}$ of light and $8 \mathrm{~h}$ of darkness. qRT-PCR analyses were performed with five biological replicates and quantification was standardized to $\beta$-TUBULIN and $\alpha$-ACTIN mRNA levels. The PCR primers used are listed in Supplementary Table S3. Statistical differences among strains or treatments were analyzed using Tukey's honestly significant difference tests in the Data Processing System software package (Tang and Zhang 2013).

\section{Targeted gene deletion and complementation.}

The deletion mutants were generated using a revised method based on a high-throughput gene knockout method described previously (Cao et al. 2016; Lu et al. 2014). A binary vector and a yeast-Escherichia-Agrobacterium shuttle vector, $\mathrm{pKO} 3 \mathrm{~A}$ and pKO3B, were constructed by replacing GFP in pKO1 or pKO1B (Lu et al. 2014) with a 1,131-bp synthesized HSVtk gene with codons optimized for $P$. oryzae using a ClonExpress MultiS One Step Cloning Kit in an in-fusion assembly method (Vazyme Biotech). HSVtk is a suicide gene encoding the herpes

Table 1. Pyricularia oryzae strains used in this study

\begin{tabular}{lll}
\hline Strain & Genotype description & Reference \\
\hline $70-15$ & Wild-type & Chao and Ellingboe 1991 \\
$\Delta m c l 1$ & $M C L 1$ deletion mutant of $70-15$ & This study \\
$\Delta m c s 1$ & $M C S 1$ deletion mutant of $70-15$ & This study \\
$\Delta m c s 1 \Delta m c l 1$ & $M C S 1$ and $M C L 1$ deletion mutant of $70-15$ & This study \\
$\Delta i c l 1$ & $I C L 1$ deletion mutant of $70-15$ & This study \\
$\Delta i c l 1 \Delta m c l 1$ & $I C L 1$ and $M C L 1$ deletion mutant of $70-15$ & This study \\
$m c l 1-c$ & $M C L 1$ rescued strain of $\Delta m c l 1$ & This study \\
$m c s 1-c$ & $M C S 1$ rescued strain of $\Delta m c s 1$ & This study \\
$\Delta i c l 1 \_m c l 1-c$ & $M C L 1$ rescued strain of $\Delta i c l 1 \Delta m c l 1$ & This study
\end{tabular}


simplex virus type 1 thymidine kinase. The construction of gene-deletion cassettes in $\mathrm{pKO} 3 \mathrm{~B}$ was performed following a previously reported method (Lu et al. 2014). Agrobacterium tumefaciens-mediated transformation (ATMT) was carried out according to a previously reported method (Lu et al. 2014), with a modification. The nitrocellulose membrane strips containing the germinated conidia cocultivated with A. tumefaciens were transferred onto the selection plates supplemented with $0.5 \mu \mathrm{M}$ 5-fluoro-2'-deoxyuridine (F2dU) and chlorimuron-ethyl at $100 \mu \mathrm{g} \mathrm{ml}^{-1}$ (for SUR, a sulfonylurea resistance gene), hygromycin B at $200 \mu \mathrm{g} \mathrm{ml}^{-1}$ (for $\mathrm{HPH}$, a hygromycin B phosphotransferase gene), or glufosinate-ammonium at $350 \mu \mathrm{g}$ $\mathrm{ml}^{-1}$ (for $B A R$, a baialaphos resistance gene). Ectopic transformants containing HSVtk could not grow on the selection media supplemented with F2dU (Khang et al. 2005). Transformants grown on the selection plates were individually transferred to the new selection plates using sterile toothpicks. Genomic DNAs of transformants were isolated and the mutants were identified and confirmed using previously reported methods ( $\mathrm{Lu}$ et al. 2014). The mutants were complemented with their respective native copy of genes amplified from the wild type. The complementation strains were identified on the selection plates supplemented with G418 at $800 \mu \mathrm{g} \mathrm{ml}^{-1}$ (for $N E O$, a neomycin phosphotransferase II gene) and confirmed at the mRNA level by RT-PCR.

\section{Observation of fluorescence fusion proteins.}

The coding sequence of $M C L 1$ was cloned from the wildtype genomic DNA and inserted into the XbaI-SalI site of pKD5-GFP containing SUR ( $\mathrm{Li}$ et al. 2012) to obtain fusion gene $G F P-M C L 1$. ATP1-RED, a fusion gene in a plasmid pKD8-RED containing $N E O$, encodes a mitochondrion marker protein (Shi et al. 2018). Both GFP-MCL1 and ATP1-RED were cotransformed into the $P$. oryzae wild-type strain via ATMT. Samples were observed under a fluorescence microscope (filter: FITC and TRITC; Nikon eclipse 80i).

\section{Phenotypic analysis of mutants.}

The growth of strains on CM, MM, and all other media was assayed at 7 to 10 days postinoculation (dpi). Conidiation on CM was analyzed at $8 \mathrm{dpi}$. The conidial germination rate was counted at 4 or 24 hpi. Appressorium turgor was evaluated through incipient cytorrhysis (cell collapse) assays, as described previously (Liu et al. 2007). The experiments were performed three times with three (for turgor test) or five (for other tests) replicates each time.

\section{Plant infections.}

Conidial virulence experiments were performed by spraying conidial suspensions $\left(1 \times 10^{5}\right.$ conidia $\left.\mathrm{ml}^{-1}\right)$ onto 10 - to 14 -dayold rice seedlings (Oryza sativa 'CO39') using an artist's airbrush (Cao et al. 2016). The disease severity was estimated by the proportion of the surface area that was diseased in a $5-\mathrm{cm}$ section of the infected leaf at $7 \mathrm{dpi}$. Mycelial virulence experiments were performed by inoculating 5-mm mycelium blocks on the leaf explants of rice or barley (Hordeum vulgare 'ZJ-8') and culturing them at $25^{\circ} \mathrm{C}$ for 4 days. Cuticle penetration experiments by appressoria on barley leaf explants and, therefore, infection growth observation were carried out according to a previously reported protocol (Liu et al. 2007).

\section{Quantification of intracellular NAD ${ }^{+}, \mathrm{NADH}$, and NO contents.}

Intracellular $\mathrm{NAD}^{+}$and NADH contents in aerial mycelia were quantified using the NAD/NADH quantification kit (Sigma-Aldrich) and NO was quantified with the NO assay kit (S0021; Beyotime) according to previously reported methods (Shi et al. 2018). The concentrations of total NAD and NADH were shown in picomoles per milligram of protein and intracellular NO in micromoles per gram of protein.

\section{Enzyme activity assays.}

The 2-methylcitrate synthase activity was measured with a citrate synthase assay kit (Sigma-Aldrich) following the manufacturer's instructions, except that acetyl-CoA from the kit was replaced with $0.3 \mathrm{mM}$ propionyl-CoA (Sigma-Aldrich). The 2-methylisocitrate lyase activity was measured using an isocitrate lyase assay kit (Solarbio) following the manufacturer's guidelines, except that isocitrate was replaced with sodium DL-threo-2-methylisocitrate (MedChem Express) at $2.5 \mathrm{mg} \mathrm{ml}^{-1}$. P. oryzae was inoculated in liquid $\mathrm{CM}$ in a rotator $(150 \mathrm{rpm})$ at $28^{\circ} \mathrm{C}$ for 2 days, then cultured in CM containing $0.002 \%$ sodium propionate for $16 \mathrm{~h}$. The mycelia were collected and ground in liquid nitrogen; then, whole-cell extracts were prepared according to the corresponding procedure in the kits. The enzyme activities were determined following the manufacturer's instructions of the kits. The 2-methylcitrate synthase and 2-methylisocitrate lyase activities were shown in microunits (nanomoles per minute) per milligram of protein.

\section{LITERATURE CITED}

Badaruddin, M., Holcombe, L. J., Wilson, R. A., Wang, Z. Y., Kershaw, M. J., and Talbot, N. J. 2013. Glycogen metabolic genes are involved in trehalose-6-phosphate synthase-mediated regulation of pathogenicity by the rice blast fungus Magnaporthe oryzae. PLoS Pathog. 9:e1003604.

Bhadauria, V., Banniza, S., Vandenberg, A., Selvaraj, G., and Wei, Y. 2012. Peroxisomal alanine: Glyoxylate aminotransferase AGT1 is indispensable for appressorium function of the rice blast pathogen, Magnaporthe oryzae. PLoS One 7:e36266.

Brock, M. 2005. Generation and phenotypic characterization of Aspergillus nidulans methylisocitrate lyase deletion mutants: Methylisocitrate inhibits growth and conidiation. Appl. Environ. Microbiol. 71:5465-5475.

Brock, M., and Buckel, W. 2004. On the mechanism of action of the antifungal agent propionate. Eur. J. Biochem. 271:3227-3241.

Brock, M., Fischer, R., Linder, D., and Buckel, W. 2000. Methylcitrate synthase from Aspergillus nidulans: Implications for propionate as an antifungal agent. Mol. Microbiol. 35:961-973.

Brock, M., Maerker, C., Schütz, A., Völker, U., and Buckel, W. 2002. Oxidation of propionate to pyruvate in Escherichia coli. Involvement of methylcitrate dehydratase and aconitase. Eur. J. Biochem. 269: 6184-6194.

Cao, H., Huang, P., Yan, Y., Shi, Y., Dong, B., Liu, X., Ye, L., Lin, F., and $\mathrm{Lu}$, J. 2018. The basic helix-loop-helix transcription factor Crf1 is required for development and pathogenicity of the rice blast fungus by regulating carbohydrate and lipid metabolism. Environ. Microbiol. 20: 3427-3441.

Cao, H., Huang, P., Zhang, L., Shi, Y., Sun, D., Yan, Y., Liu, X., Dong, B., Chen, G., Snyder, J. H., Lin, F., and Lu, J. 2016. Characterization of 47 Cys2 -His2 zinc finger proteins required for the development and pathogenicity of the rice blast fungus Magnaporthe oryzae. New Phytol. 211:1035-1051.

Chao, C. C. T., and Ellingboe, A. H. 1991. Selection for mating competence in Magnaporthe grisea pathogenic to rice. Can. J. Bot. 69:2130-2134.

Cheema-Dhadli, S., Leznoff, C. C., and Halperin, M. L. 1975. Effect of 2methylcitrate on citrate metabolism: Implications for the management of patients with propionic acidemia and methylmalonic aciduria. Pediatr. Res. 9:905-908.

Dean, R., Van Kan, J. A., Pretorius, Z. A., Hammond-Kosack, K. E., Di Pietro, A., Spanu, P. D., Rudd, J. J., Dickman, M., Kahmann, R., Ellis, J., and Foster, G. D. 2012. The Top 10 fungal pathogens in molecular plant pathology. Mol. Plant Pathol. 13:414-430.

de Jong, J. C., McCormack, B. J., Smirnoff, N., and Talbot, N. J. 1997 Glycerol generates turgor in rice blast. Nature 389:244.

Dubey, M. K., Broberg, A., Jensen, D. F., and Karlsson, M. 2013. Role of the methylcitrate cycle in growth, antagonism and induction of systemic defence responses in the fungal biocontrol agent Trichoderma atroviride. Microbiology 159:2492-2500.

Elliot, M. A., and Talbot, N. J. 2004. Building filaments in the air: Aerial morphogenesis in bacteria and fungi. Curr. Opin. Microbiol. 7:594-601.

Fernandez, J., Marroquin-Guzman, M., and Wilson, R. A. 2014. Evidence for a transketolase-mediated metabolic checkpoint governing biotrophic 
growth in rice cells by the blast fungus Magnaporthe oryzae. PLoS Pathog. 10:e1004354.

Fernandez, J., Wright, J. D., Hartline, D., Quispe, C. F., Madayiputhiya, N., and Wilson, R. A. 2012. Principles of carbon catabolite repression in the rice blast fungus: Tps1, Nmr1-3, and a MATE-family pump regulate glucose metabolism during infection. PLoS Genet. 8:e1002673.

Fleck, C. B., and Brock, M. 2008. Characterization of an acyl-CoA: Carboxylate CoA-transferase from Aspergillus nidulans involved in propionyl-CoA detoxification. Mol. Microbiol. 68:642-656.

Graham, I. A., and Eastmond, P. J. 2002. Pathways of straight and branched chain fatty acid catabolism in higher plants. Prog. Lipid Res. 41: 156-181.

Halarnkar, P. P., and Blomquist, G. J. 1989. Comparative aspects of propionate metabolism. Comp. Biochem. Physiol. Part B Biochem Mol. Biol. 92:227-231.

Horswill, A. R., Dudding, A. R., and Escalante-Semerena, J. C. 2001. Studies of propionate toxicity in Salmonella enterica identify 2methylcitrate as a potent inhibitor of cell growth. J. Biol. Chem. 276: 19094-19101.

Ibrahim-Granet, O., Dubourdeau, M., Latgé, J. P., Ave, P., Huerre, M., Brakhage, A. A., and Brock, M. 2008. Methylcitrate synthase from Aspergillus fumigatus is essential for manifestation of invasive aspergillosis. Cell. Microbiol. 10:134-148.

Khang, C. H., Park, S. Y., Lee, Y. H., and Kang, S. 2005. A dual selection based, targeted gene replacement tool for Magnaporthe grisea and Fusarium oxysporum. Fungal Genet. Biol. 42:483-492.

Lee, S. H., Han, Y. K., Yun, S. H., and Lee, Y. W. 2009. Roles of the glyoxylate and methylcitrate cycles in sexual development and virulence in the cereal pathogen Gibberella zeae. Eukaryot. Cell 8:1155-1164.

Li, H. J., Lu, J. P., Liu, X. H., Zhang, L. L., and Lin, F. C. 2012. Vectors building and usage for gene knockout, protein expression and fluorescent fusion protein in the rice blast fungus. J. Agric. Biotechnol. 20:94-104.

Limenitakis, J., Oppenheim, R. D., Creek, D. J., Foth, B. J., Barrett, M. P., and Soldati-Favre, D. 2013. The 2-methylcitrate cycle is implicated in the detoxification of propionate in Toxoplasma gondii. Mol. Microbiol. 87:894-908.

Liu, X. H., Lu, J. P., Zhang, L., Dong, B., Min, H., and Lin, F. C. 2007. Involvement of a Magnaporthe grisea serine/threonine kinase gene, $M g A T G 1$, in appressorium turgor and pathogenesis. Eukaryot. Cell 6: 997-1005.

Lu, J., Cao, H., Zhang, L., Huang, P., and Lin, F. 2014. Systematic analysis of Zn2Cys6 transcription factors required for development and pathogenicity by high-throughput gene knockout in the rice blast fungus. PLoS Pathog. 10:e1004432.

Luttik, M. A., Kötter, P., Salomons, F. A., van der Klei, I. J., van Dijken, J. P., and Pronk, J. T. 2000. The Saccharomyces cerevisiae ICL2 gene encodes a mitochondrial 2-methylisocitrate lyase involved in propionylcoenzyme A metabolism. J. Bacteriol. 182:7007-7013.

Maerker, C., Rohde, M., Brakhage, A. A., and Brock, M. 2005. Methylcitrate synthase from Aspergillus fumigatus. Propionyl-CoA affects polyketide synthesis, growth and morphology of conidia. FEBS J. 272:3615-3630.

Müller, S., Fleck, C. B., Wilson, D., Hummert, C., Hube, B., and Brock, M. 2011. Gene acquisition, duplication and metabolic specification: The evolution of fungal methylisocitrate lyases. Environ. Microbiol. 13:1534-1548.
Norvienyeku, J., Zhong, Z., Lin, L., Dang, X., Chen, M., Lin, X., Zhang, H., Anjago, W. M., Lin, L., Abdul, W., and Wang, Z. 2017. Methylmalonatesemialdehyde dehydrogenase mediated metabolite homeostasis essentially regulate conidiation, polarized germination and pathogenesis in Magnaporthe oryzae. Environ. Microbiol. 19:4256-4277.

Papanikolaou, S., Beopoulos, A., Koletti, A., Thevenieau, F., Koutinas, A. A., Nicaud, J. M., and Aggelis, G. 2013. Importance of the methylcitrate cycle on glycerol metabolism in the yeast Yarrowia lipolytica. J. Biotechnol. 168:303-314.

Peyraud, R., Kiefer, P., Christen, P., Massou, S., Portais, J. C., and Vorholt, J. A. 2009. Demonstration of the ethylmalonyl-CoA pathway by using 13C metabolomics. Proc. Natl. Acad. Sci. U.S.A. 106:4846-4851.

Pronk, J. T., van der Linden-Beuman, A., Verduyn, C., Scheffers, W. A., and van Dijken, J. P. 1994. Propionate metabolism in Saccharomyces cerevisiae: Implications for the metabolon hypothesis. Microbiology 140:717-722

Schwab, M. A., Sauer, S. W., Okun, J. G., Nijtmans, L. G., Rodenburg, R. J., van den Heuvel, L. P., Dröse, S., Brandt, U., Hoffmann, G. F., Ter Laak, H., Kölker, S., and Smeitink, J. A. 2006. Secondary mitochondrial dysfunction in propionic aciduria: A pathogenic role for endogenous mitochondrial toxins. Biochem. J. 398:107-112.

Shi, Y., Wang, H., Yan, Y., Cao, H., Liu, X., Lin, F., and Lu, J. 2018. Glycerol-3-Phosphate shuttle is involved in development and virulence in the rice blast fungus Pyricularia oryzae. Front. Plant Sci. 9:687.

Talbot, N. J., Ebbole, D. J., and Hamer, J. E. 1993. Identification and characterization of $M P G 1$, a gene involved in pathogenicity from the rice blast fungus Magnaporthe grisea. Plant Cell 5:1575-1590.

Tang, Q. Y., and Zhang, C. X. 2013. Data Processing System (DPS) software with experimental design, statistical analysis and data mining developed for use in entomological research. Insect Sci. 20: 254-260.

Thines, E., Weber, R. W. S., and Talbot, N. J. 2000. MAP kinase and protein kinase A-dependent mobilization of triacylglycerol and glycogen during appressorium turgor generation by Magnaporthe grisea. Plant Cell 12: 1703-1718.

Uchiyama, H., Ando, M., Toyonaka, Y., and Tabuchi, T. 1982. Subcellular localization of the methylcitric-acid-cycle enzymes in propionate metabolism of Yarrowia lipolytica. Eur. J. Biochem. 125:523-527.

Wang, Z. Y., Soanes, D. M., Kershaw, M. J., and Talbot, N. J. 2007. Functional analysis of lipid metabolism in Magnaporthe grisea reveals a requirement for peroxisomal fatty acid beta-oxidation during appressorium-mediated plant infection. Mol. Plant-Microbe Interact. 20:475-491.

Wang, Z. Y., Thornton, C. R., Kershaw, M. J., Debao, L., and Talbot, N. J. 2003. The glyoxylate cycle is required for temporal regulation of virulence by the plant pathogenic fungus Magnaporthe grisea. Mol. Microbiol. 47:1601-1612.

Zhang, Y., Shi, H., Liang, S., Ning, G., Xu, N., Lu, J., Liu, X., and Lin, F. 2015. MoARG1, MoARG5,6 and MoARG7 involved in arginine biosynthesis are essential for growth, conidiogenesis, sexual reproduction, and pathogenicity in Magnaporthe oryzae. Microbiol. Res. 180: 11-22.

Zhang, Y. Q., Brock, M., and Keller, N. P. 2004. Connection of propionylCoA metabolism to polyketide biosynthesis in Aspergillus nidulans. Genetics 168:785-794. 